\title{
Meso-scale modelling of static and dynamic tensile fracture of concrete accounting for real-
}

\section{shape aggregates}

\author{
Sadjad Naderi, Mingzhong Zhang* \\ Department of Civil, Environmental and Geomatic Engineering, University College London, \\ London, WC1E 6BT, UK
}

\begin{abstract}
This paper presents a computational framework for modelling the fracture process in concrete under static and dynamic tensile loading considering its mesostructural characteristics. 3D mesostructure of concrete composed of real-shape coarse aggregates, mortar, interfacial transition zone between them and voids was developed using an in-house code based on Voronoi tessellation and splining techniques. Cohesive zone model was then employed to simulate the tensile fracture behaviour of concrete in terms of stress- and energy dissipation-displacement responses and crack mechanisms against the shape (spherical and irregular) and volume fraction (30\%, 35\% and 40\%) of aggregate and strain rate $\left(0,1,10\right.$ and $\left.50 \mathrm{~s}^{-1}\right)$. Results indicate that the irregular shape of aggregate has an important role in the micro-crack nucleation and ultimate fracture pattern but exhibits an insignificant effect on the tensile strength of concrete, which is mainly dependent on the strain rate and the random location and size distribution of aggregate.
\end{abstract}

Keywords: Mesostructure; Irregular shape aggregate; Cohesive element; Damage evolution; Finite element analysis

\section{Introduction}

Concrete as one of the most important construction materials may inevitably experience tensile failure under different loading conditions in events like earthquakes and explosions during service life [1]. Such failure becomes even more pronounced in tension due to the low tensile strength of normal concrete. Considering this fact, it is necessary to provide knowledge for better structural design against the failure in terms of safety, serviceability and durability [2-4]. Therefore, a number of studies have been focused on static and dynamic tensile fracture behaviour of concrete over the last decades, which continues to be a topic of high importance for the accurate prediction of concrete performance under service load and aggressive environment [5-7]. To cover all aspects of the damage mechanism, it is vital to gain a comprehensive understanding of not only stress-strain response [ $\underline{8}]$ but also fracture

\footnotetext{
* Corresponding author. E-mail address: mingzhong.zhang@ucl.ac.uk (M. Zhang)
} 
process and crack morphology [9-13], which are highly associated with overall mechanical strengths and transport properties of concrete and thus the long-term durability of concrete infrastructure [14].

Until now, many experimental efforts have been made to investigate the stress-displacement response and fracture toughness of concrete [15]. However, it is still a big challenge to accurately detect the local stress and crack initiation and propagation inside a concrete sample during loading, which play a crucial role in interpreting in mechanical performance of concrete [16-18]. It can become more problematic if a test is performed under high strain loading due to the fast growth of the cracks. To tackle this challenge, the numerical approaches have been increasingly adopted rather than analytical frameworks for such high-heterogeneous composite. Among the proposed numerical methods, mesostructure-based models have been drawing more attention as they enable to incorporate the internal structural features, which would significantly influence the fracture process in concrete $[\underline{19}, \underline{20}]$. These models are generally constructed by coarse aggregates randomly distributed in a homogenous mortar with interfacial transition zones (ITZs) between them and also observable voids at meso-level [21] , in which the heterogenous nature of concrete can be considered to investigate the effect of different phases on the macroscopic mechanical behaviour and crack initiation and propagation of concrete [22]. The reliability of numerical results obtained from such models is tied to the aggregate structure, which governs the mechanical response and crack morphology. It was reported that the location, size and local shape properties of aggregates play an important role in regional and global stress field that significantly influences the fracture mechanism of concrete under different loading conditions [23-25], particularly in a dynamic regime, where its role can become even more dominant under high-rate loading [26]. So, for the sake of accuracy, coarse aggregates should be comparable with experimental data in terms of size and shape distribution. Most of the previous computer-based models could simulate the random location of aggregates and their size distribution obtained from the experiments $[\underline{27}, \underline{28}]$, however, the main concern is raised over the aggregate shape and the simulation efficiency. To clearly state the major issues, different modelling approaches will be discussed below.

A mesostructure of concrete can be generally modelled using image-dependent and imageindependent approaches [28]. In comparison, an image-based method can generate a mesostructure with structural properties closer to reality (e.g. using high resolution images obtained from X-ray computed tomography scans [29]). However, the capability of this approach is restricted to the 
specific image data and computational cost $[\underline{8}, \underline{30}, \underline{31}]$. The method can yield a limited number of mesostructure models, which may be insufficient for a statistical-based characterisation and it is not very adjustable with respect to some parameters like aggregate size/shape distribution. Its efficiency was improved in some cases, but the algorithms still suffered from the expensive computational process. For instance, some representative aggregates were initially replicated as many as required and then randomly placed in the model domain [32]. Such method relies on the complex image reconstruction techniques and encounters the overlapping problem that is a common matter in the simulation of a random system. As an inexpensive alternative, image-independent methods can be used owing to higher efficiency and better adjustability [33], where aggregate models are normally simplified by three geometries $[\underline{8}, \underline{34}, \underline{35}]$. The first is a perfect sphere, which does not reflect any shape characteristics of actual aggregates. However, they might be useful in simulation of some fine aggregates like natural gravels, which have semi-spherical/rounded geometry [ㅈ6]. The second is ellipsoid to model the effect of the aspect ratio but it is not capable to address the angularity of aggregate surfaces. The sharp corners and edges as undeniable features of an actual aggregate are important since they can considerably increase the local stress concentration and consequently influence the damage regime. The third is polyhedron to model the irregularity of aggregates but their geometric properties might not be consistent with the reality very well. In contrast with extreme roundness of spherical and ellipsoidal structures, the high angularity of polyhedron geometry would increase the stress concentration more than the expectation. In addition, the relatively large flatten face can lead to underestimating stress level. Although, like the spherical model, the polyhedrons can be assumed as an idealized geometry of some aggregates, e.g. crushed rocks [37]. Therefore, there is a strong need for a better model to guarantee the shape effect of the real aggregates on mechanical behaviour of concrete with a reasonable computational cost.

To tackle the above-mentioned limitations and issues, this study aims to develop a new computational modelling framework for an in-depth, accurate analysis of static and dynamic tensile fracture process in concrete in terms of stress-displacement response, dissipated energy and crack morphology. The framework mainly consists of an advanced cost-effective mesostructure model of concrete and a cohesive zone model (CZM). The three-dimensional (3D) mesostructure of concrete containing irregular aggregates with realistic shape characteristics is generated using a robust algorithm developed in the previous work [28] through the implementation of Voronoi tessellation 
and splining techniques. Regarding model adjustability, the notable features enable to quantitively control the shape and size distribution of aggregates with reference to empirical data in the form of shape parameters and sieve analysis, respectively. Owing to model measurability, the triangulated surface representation of the aggregates allows to precisely compute the shape parameters, which can be reported in two levels of accuracy. The first describes overall shape by sphericity and elongation ratio. The second indicates local shape by roundness and convexity. These parameters of the simulated aggregates with various shapes are compared and validated with those experimentally acquired for the typical aggregates used in conventional concrete. The generated virtual 3D mesostructure of concrete is then incorporated into finite element $(\mathrm{FE})$ simulations of fracture process in concrete using CZM, which provides more natural crack representation. CZM becomes more and more popular for modelling cracks because of its easy implementation and simple formation in terms of cohesive

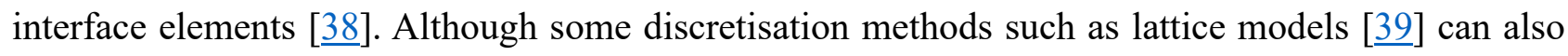
provide the realistic simulation of fracture behaviour of concrete, the main issue is related to the difficulty in the equivalent description of the continuum properties, and such equivalent description is difficult to generalize for different stress conditions $[\underline{40}, \underline{41}]$. The determination of equivalent model parameters is relatively straightforward for CZM as a continuum-based FE model [42]. The highquality visualization of the simulated cracks is rendered to provide the best conditions for qualitative evaluation whereas illuminating some aspects that might be hidden from view using the traditional methods.

To demonstrate the potential of this new modelling framework for understanding the relationship between mesostructure and fracture behaviour in concrete, the effects of structural variables (e.g. aggregate shape, size and content) and strain rate $\left(0,1,10\right.$ and $\left.50 \mathrm{~s}^{-1}\right)$ on fracture process in concrete under uniaxial static and dynamic tensile loads are investigated here. First, a three-phase geometry model of concrete composed of irregularly shaped coarse aggregates, mortar and voids is generated using an in-house package coded in MATLAB [28]. Then, the zero-thickness cohesive elements that represent the ITZs between aggregates and mortar as the fourth phase in concrete are created and embedded into continuum tetrahedral elements of mortar. ITZ is a thin layer surrounding each aggregate and is normally considered as the weak bound in concrete due to the relatively high waterto-cement ratio and porosity in this region [43]. Afterwards, the generated mesostructure models of concrete are exported to ABAQUS for the FE explicit simulations. Some important parameters 
including the element size, the static-loading time and the sample numbers are set up according to a previous study []], where the mesostructure models of concrete with regularly shaped (i.e. spherical, ellipsoidal and polyhedral) aggregates were developed and comprehensively studied. To understand the effect of geometric simplifications, a mesostructure with spherical aggregates is modelled with the same size distribution using the algorithm that has been repeatedly described in the literature $[\underline{18}$, 44-46]. A comparison between the models with real-looking and spherical aggregates highlights the advantage of the current method against the conventional approaches. Moreover, the mesostructure models of concrete with $30 \%, 35 \%$ and $40 \%$ aggregates and $2 \%$ voids are used to estimate the influence of aggregate content on tensile fracture of concrete under different strain rates.

\section{3D mesostructure of concrete}

In the first step, the surface of aggregates and the voids are constructed by the triangle elements in a cubic representative volume element (RVE) using two types of geometry, including spheres and irregular splined Voronoi cells [28] labelled as $\boldsymbol{S P H}$ and $\boldsymbol{I R G}$, respectively. For the model IRG, the splining process is mainly used to convert the angular Voronoi cells to a structure, which is more like the natural aggregates. In practice, the sharp edges and corners of the polyhedral cells are regularised into more rounded features. In other terms, it transforms the flat faces to the curved ones and decreases the high angularity. Fig. 1 schematically shows the transformation of the original Voronoi cell to the splined Voronoi morphology. It is worth pointing out that the splining technique is based on subdividing surfaces and it can be performed in multi-subdivision iterations. A smoother surface is produced after each iteration level. Here, two iteration levels are applied. In the following sections, the shape and size configurations of the aggregates are reported to demonstrate the geometric similarity to the reality from a reliability point of view. This paper is not concerned with the shape properties of the void due to its low volume fraction and the small size. However, in practice, it can be said that the voids have structures like the coarse aggregates. For visual comparison, the examples of two types of structures are illustrated in Fig. $2 a$ and b. In order to achieve a reportative volume, the side length of the RVE is adjusted to $50 \mathrm{~mm}$ to ensure it is at least three to five times greater than the size of the largest aggregates [34].

In the second step, a non-uniform mesh generation method is applied to fill the inner regions enclosed by the constructed triangulated surfaces of the aggregates using irregular tetrahedral elements. Then, the available space among the outer surfaces of the aggregates, the voids and the 
RVE walls is meshed to constitute the mortar. The proposed mesh technique showed a reasonable computational cost for such a heterogeneous structure. In the non-uniform mesh generation, the maximum element characteristic length is adjusted to $1 \mathrm{~mm}$ as adopted in [] based on the element size sensitivity analysis in order to achieve a balance between the accuracy and the computational efficiency. Here, the number of tetrahedral element ranges from 1.3 to 1.6 million for all models.

The final step of the mesostructure modelling process is the insertion of the cohesive elements using the developed in-house code based on the nodal and elemental data. The corresponding algorithm will be described in Section 3.1. The main steps for generating the 3D mesostructure of concrete are summarised in the process flow diagram (Fig. 3) with the order of the phase formation.

\subsection{Shape properties of aggregates}

For an accurate characterisation, several shape descriptors are required for irregular geometries of aggregates in the model IRG. Here, four shape parameters are used, which indicate the general and local deviation from the surface of the perfect sphere. Sphericity and elongation ratio are used as the global shape descriptors, while roundness and convexity describe the local shape of the surface. It should be noted that in terms of irregularity definition and shape parameters calculation, there is no set of criteria or widely accepted method. It may be problematic for comparison with other empirical studies. For instance, circularity is considered instead of sphericity in a two-dimensional (2D) framework. However, the results can be somehow compared with each other when the shape parameters attained from the experiments are the only way to validate the model and all approaches intend to deliver the same geometric meaning. In this study, the equations below are adopted to compute the parameters using the triangle elements that create the cell surface [28]:

$$
\begin{aligned}
& \text { Sphericity }=\frac{\text { Surface Area of Equivalent Sphere }}{\text { Surface Area of Particle }}=\frac{4 \pi\left(\frac{3 V}{4 \pi}\right)^{2 / 3}}{S} \\
& \text { Elongation ratio }=\frac{L_{\min }}{L_{\max }} \\
& \text { Roundness }=\frac{\sum_{i=1}^{m}\left|\hat{r}_{i} \cdot \hat{n}_{i}\right| \triangle S_{i}}{S} \\
& \text { Convexity }=\frac{V}{V_{C H}}
\end{aligned}
$$

where $V$ and $S$ denote the volume and surface area, respectively, $L$ stands for the distance between the centre of the cell and a node on the surface, $\hat{r}_{i} \cdot \hat{n}_{i}$ gives the angle between a vector from cell centroid to centre of the $i$-th triangle element and the corresponding normal vector of the element, $\triangle S_{i}$ 
represents the area of the element, $m$ is the total number of elements for the cell, and $V_{C H}$ is the volume of convex hull computed by the function of convhull in MATLAB. All these parameters vary from zero to one (for a perfect sphere).

In order to obtain the shape parameter distribution among all the cells in the model $I R \boldsymbol{G}$, a module is incorporated into the main MATLAB code to produce a histogram for each model. It should be mentioned that 30 random samples can yield per model set to produce faithful results from a statistical point of view. To achieve a representative histogram for each set, first, the whole range of values should be divided into the same series of intervals (or bins) for all samples in a set; second, it is counted how many values fall into each interval per sample; third, a mean value per interval is calculated by averaging the data in the corresponding interval for all models. Fig. $2 \mathrm{~d}$ shows the representative histogram of the shape parameters for all concrete samples with $30 \%$ irregular aggregates. The standard deviation for each bin is less than 0.1 for all plots, which proves that the number of models is enough in terms of shape. The sufficiency of the number of samples regarding the mechanical properties will be discussed in detail in Section 4.

By comparing the aggregate shape between the virtual and real mesostructure of concrete, it can be concluded that the simulated ranges of shape parameters are mostly consistent with the experimental data acquired for the common aggregates. The sphericity value of natural gravels and crushed rocks ranges from 0.6 to 0.9 [47-49], which is almost equivalent to the range presented in Fig. $2 \mathrm{~d}(0.65$ to 0.95$)$. Likewise, the roundness range (0.55 to 0.95$)$ agrees well with the experimental data $(0.6$ to 0.8$)$ [49]. The elongation ratio changes in a relatively wider range in both the model $(0.15$ to 0.6$)$ and the experiments ( 0.3 to 0.7$)$ [47]. The splining technique enables to simulate the convexity within a range of 0.95 to 0.99 , which is slightly larger than the measured data $(0.85$ to 0.95$)$ [ $\underline{50}, \underline{51}$.

\subsection{Size distribution of aggregates and voids}

In the sieve analysis, the mass fraction of particles is measured within each size range to compute the particle size distribution of aggregates, which can be described in the form of the grading curves such as the Fuller curve [28]. The design of aggregate grading based on the Fuller curve leads to an optimum concrete mixture, which can be expressed as []]:

$$
P(D)=100\left(\frac{D}{D_{\max }}\right)^{n}
$$

where $P(D)$ denotes the cumulative percentage passing a sieve with aperture diameter $D, D_{\max }$ is 
the maximum size of aggregates, and $n$ is the exponent of the equation that normally equals to 0.5 .

For the easy implementation of the gradation curve in a numerical format, it can be segmented, where the amount of aggregates $V_{a g g}$ within each grading segment $\left[D_{i}, D_{i+1}\right]$ can be obtained by:

$$
V_{a g g}\left[D_{i}, D_{i+1}\right]=\frac{P\left(D_{i+1}\right)-P\left(D_{i}\right)}{P\left(D_{\max }\right)-P\left(D_{\min }\right)} \times P_{a g g} \times V_{\text {total }}
$$

where $D_{\min }$ represents the minimum aggregate size, $P_{a g g}$ is the volume fraction of aggregates, and $V_{\text {total }}$ denotes the total volume of concrete specimen.

A common particle size distribution of aggregates with four sieve groups [52] as given in Table 1 is used in the aggregate size adjustment, which groups the coarse aggregates into three size ranges including small (2.36 to $4.75 \mathrm{~mm}$ ), medium (4.75 to $9.5 \mathrm{~mm}$ ) and large ( 9.5 to $12.7 \mathrm{~mm}$ ). Here, the size of air voids as mesoscopic features [두] is considered to be ranging from 2 to $4 \mathrm{~mm}$ [8]. The calibration process is performed to adjust aggregates and voids using the algorithm presented in [28]. Although, before the adjustment process, the minimum distance between two aggregates was set greater than the double maximum thickness of ITZ $(0.05 \mathrm{~mm})$ measured in the experiments [43] in order to be consistent with the geometric dimensions of the real mesostructure. It is assumed that the aggregates occupy $30 \%$ of the total volume of concrete, while the RVE of concrete contains $2 \%$ voids. Fig. $2 \mathrm{a}$ and $\mathrm{b}$ show the simulated mesostructure models of concrete containing spherical aggregates and irregular aggregates respectively, i.e. SPH and $\boldsymbol{I R G}$. The size segmentations of aggregates and voids are accordingly specified by different colours for both the models $\boldsymbol{I R \boldsymbol { G }}$ and $\boldsymbol{S P H}$ and illustrated in Fig. 2c. To investigate the effect of aggregate volume fraction, two more sets of the model IRG with aggregate content of $35 \%$ and $40 \%$ are generated with the same content of voids in concrete. Like the shape distribution, the mean values of the aggregate probability density in each size range are obtained for the model $\boldsymbol{I R \boldsymbol { G }}$ with 30\% aggregates and displayed in Fig. 2c and d.

\section{Cohesive zone modelling}

\subsection{Cohesive element insertion}

Beforehand, the model comprised of aggregates, voids and mortar is meshed by tetrahedral elements. The output file generated contains the array data on nodes and elements. All nodes and elements should be numbered consecutively to ease programming in this stage. Each element is tagged by the material identification (ID) number, i.e. 1 for mortar and 2 for aggregates. Such file is applied as an input to a MATLAB code to embed the cohesive elements at the interface between two adjacent 
tetrahedral elements in the initial mesh structure. The proposed algorithm follows the following main steps (Fig. 3):

- The input array data is read and converted into two matrices designated for nodes and elements, which have three and five columns, respectively. Row number is considered as the node and element number. The nodes matrix includes nodal coordinates in the Cartesian system while the element matrix contains the nodal connectivity based on the node numbers in the first-fourth column and the material ID number in the fifth column. It is very important to correctly define an element orientation by the right sequence of node number as exemplified in Fig. 4a for a tetrahedral element.

- For each element, the adjacent elements need to be specified based on shared nodes and then saved in a matrix. For this, a useful MATLAB function, named "neighbors()", is employed.

- For each element, the coordinates of the nodes are duplicated from the original node matrix and sequentially added into a new matrix. Consequently, the node numbering is changed and therefore, the nodal connectivity should be accordingly updated. In practice, this operation detaches the elements from each other, which are used to be connected in the initial continuous mesh.

- The new matrices obtained from the two previous steps are used to generate the so-called cohesive interface element (CIE) as a 6-node triangular prism element with zero thickness (Fig. 4a). The method to share the nodes between the CIE and the mutual faces of two neighbour tetrahedron elements is schematically shown in Fig. 4b. After this step, all the tetrahedral elements are reconnected through the CIEs. It should be mentioned that the orientation of CIEs must be consistent with the orientation of tetrahedron elements for the right connection in the finite element mesh structure. The CIEs generated need to be labelled with a material ID number in the array data. The nodal connectivity of the CIEs is saved in a matrix with seven columns. In this study, CIE-AGG, CIE-MOR and CIE-INT are used for the elements inserted into aggregateaggregate, mortar-mortar and aggregate-mortar interfaces, respectively.

- The matrices of the duplicated nodes, the updated tetrahedron elements and the generated CIEs are employed to yield an input file to ABAQUS with INP format. Regarding the element types, C3D4 and COH3D6 in ABAQUS are used for tetrahedral and cohesive elements, respectively. The element sets are made based on the element types and the material IDs number. As an example, Fig. 5 separately shows the element sets that compose the final version of the model. 
In addition to the mesh properties, the INP file contains information about material properties, boundary conditions, initial and loading conditions, which will be described in the following sections.

\subsection{Material model}

In normal concrete, the aggregates have a much higher tensile strength in comparison with mortar and ITZs [54]. Therefore, it is acceptable to assume that aggregates behave like a damage-free elastic material while the crack initiates and develops in ITZ and mortar as the weaker constituents. To implement this assumption in the FE cohesive zone model, the elastic behaviour is assigned to all the tetrahedral elements and the CIE-AGG whereas the damage variables are only defined for the CIEMOR and the CIE-INT. The theory related to the constitutive response of cohesive elements has been well documented in ABAQUS user Manual and frequently explained in the literature [ $\underline{8}, \underline{55}]$. Thus, the key model configurations are merely pointed out in this section.

Regarding the cohesive element sets, the linear elastic traction-separation relationship is adopted with the ABAQUS keyword *ELASTIC, TYPE=TRACTION. For the CIE-MOR and CIE-INT, the initial elastic behaviour assumed is followed by the initiation and linear evolution of damage based on the quadratic nominal stress criterion and fracture energy, respectively. It means that a crack nucleates when the quadratic interaction function was fulfilled as follows:

$$
\left\{\frac{\left\langle t_{n}\right\rangle}{t_{n 0}}\right\}^{2}+\left\{\frac{t_{s}}{t_{s 0}}\right\}^{2}+\left\{\frac{t_{t}}{t_{t 0}}\right\}^{2}=1
$$

where $t_{n}, t_{s}$ and $t_{t}$ denote the normal traction and two tangential tractions (shear cohesion), respectively, $t_{n 0}, t_{s 0}$ and $t_{t 0}$ are the critical tractions for three modes, and \langle\rangle is Macaulay bracket:

$\langle x\rangle=\left\{\begin{array}{lr}x, \quad x \geq 0 \text { (Tension) } \\ 0, \quad x<0 \text { (Compression) }\end{array}\right.$

In addition to tensile/shear strength, $t_{0}$, the normal/tangential fracture energy, $G$, is specified to fully determine the bilinear traction-separation law. Hence, the failure separation, $\delta_{f}$, can be calculated according to:

$$
G=\int_{0}^{\delta_{f}} t(\delta) d \delta=\frac{1}{2} t_{0} \delta_{f}
$$

To describe the linear softening evolution under the combined normal and tangential separation, the effective relative displacement, $\delta_{m}$, and scalar damage, $D$, are used as expressed below:

$$
\delta_{m}=\sqrt{\left\langle\delta_{n}\right\rangle^{2}+\delta_{s}^{2}+\delta_{t}^{2}}
$$




$$
D=\frac{\delta_{m f}\left(\delta_{m, \max }-\delta_{m 0}\right)}{\delta_{m, \max }\left(\delta_{m f}-\delta_{m 0}\right)}
$$

where $\delta_{m, \max }$ represents the maximum effective relative displacement obtained during the loading history, $\delta_{m 0}$ and $\delta_{m f}$ denote the effective displacement at damage initiation and final failure, respectively, and the variable $D$ evolves monotonically from 0 (intact) to 1 (fully damage).

The stress components of the traction-separation model are affected by the damage as follows:

$$
\begin{aligned}
t_{n} & = \begin{cases}(1-D) \bar{t}_{n}, & \bar{t}_{n} \geq 0 \\
\bar{t}_{n}, & \bar{t}_{n}<0 \text { (no damage to compressive stiffness) }\end{cases} \\
t_{s} & =(1-D) \bar{t}_{s}, t_{t}=(1-D) \bar{t}_{t}
\end{aligned}
$$

where $\bar{t}_{n}, \bar{t}_{s}$ and $\bar{t}_{t}$ are the traction components predicted by the elastic traction-displacement behaviour for the current separation without damage.

To set these, the keywords *DAMAGE INITIATION, CRITERION=QUADS and *DAMAGE EVOLUTION, TYPE=ENERGY are used. As an output variable, the index of SDEG is selected to determine the overall damage. Regarding the tetrahedral elements, the elastic modulus and Poisson's ratio are adjusted for mortar and aggregates. Table 2 lists the corresponding material properties defined in the current model, which are validated with other studies and experiments in the previous work [ $\underline{8}$. The initial elastic stiffness needs to be in the range of 20-50 times greater than the elastic modulus of solid element for bulk material with Poisson's ratio of 0.2 and average cohesive element size of $1 \mathrm{~mm}[\underline{56}, \underline{57}]$. A large enough value of stiffness allows avoiding artificial compliance, which would change the dynamic wave speed. On the other hand, the cohesive stiffness does not need to be extremely high because it would unnecessarily reduce the time step.

It should be noted that the constitutive laws of the CIEs proposed are simplified in two ways. First, due to lack of accurate experimental data, the shear fracture properties were assumed to be as same as those in normal direction. Also, the shear stress and normal stress are not fully coupled, which would be more important in compression mode [58]. Consequently, the model is not able to accurately simulate cracking induced by shear failure. However, it was demonstrated that this simplification has a minor influence on the analysis of concrete under uniaxial tensile loading based on the validation of numerical results in [ㅁ] and two-dimensional parametric studies in [59]. Second, the material properties are assumed to be rate-independent as reported in $[60,61]$ and the effects of other parameters, e.g. the viscous behaviour of the mortar matrix, are not considered. In the high strain rate loading regime $(\dot{\varepsilon}>1)$, the dynamic tensile strength and fracture energy of concrete are enhanced 
[62]. However, this study aims to only explore the effect of structural features at mesoscale, which mainly influence the mechanical response [21], and the estimation of dynamic increase factor for concrete is beyond the scope of this study.

\subsection{Loading and boundary conditions}

For static loading, the uniaxial displacement-control method is considered. One face of the RVE is subjected to the vertical displacement while its opposite face is fixed (Fig. 6). The simulation is terminated at a maximum prescribed displacement of $0.1 \mathrm{~mm}$ to enable the analysis of the data up to which most of the damage occurred in the specimen. The displacement gradually increases using a smooth step amplitude curve and with a long enough time of $0.01 \mathrm{~s}$. It allows simulating the quasistatic conditions by reducing the kinetic energy, increasing stability and ensuring convergence. For this, the ratio of the total kinetics to the total internal energy was checked to be always below $5 \%$ during the simulation time [63]. Besides, the convergence test regarding the loading time was carried out through the Monte Carlo analysis of the stress-displacement curves and the crack patterns using the models with $30 \%$ spherical aggregates in the previous work []], which indicated that the acceptable minimum loading time is $0.005 \mathrm{~s}$ considering the computational efficiency and accuracy.

Regarding dynamic loading, in the same way, the sample is loaded under a displacementcontrolled system but with an imposed strain rate $\dot{\varepsilon}$, which is set to 1,10 and $50 \mathrm{~s}^{-1}$ corresponding to low-moderate and high strain rates. To apply this, all the nodes located on two opposite faces of the RVE are constrained to vertically move in the opposite directions as illustrated in Fig. 6 with a constant velocity $V_{0}$ that is related to the side length of RVE $h$ as follows:

$$
V_{0}=\dot{\varepsilon} \frac{h}{2}
$$

To avoid the stress propagation and an early fracture near the boundaries, an initial velocity profile is applied to all the nodes as a function of the vertical position [64]:

$$
V_{y}(y, t=0)=\frac{2 V_{0}}{h} y
$$

where $y=0$ is the mid-plane of the RVE.

It is worth noting that the exact linear gradient might not be achieved by the numerical implementation of the above equation into the model through the nodes non-uniformly distributed. Although, the initial conditions proposed do not lead to oscillations at the early stage of the loading. 
In such FE problem, the stability is achieved in the numerical explicit solution when the time step is smaller than the following critical value [64]:

$$
\Delta t_{c r i t}=\alpha \min _{e=1 \ldots N}\left(\frac{l_{e}}{c}\right)
$$

where $c$ represents the compressive stress wave speed, $l_{e}$ is the characteristic length of $\mathrm{e}^{\text {th }}$ elements, $N$ is the total number of elements, and $\alpha$ is a security coefficient (typically around 0.1 ).

In ABAQUS, an explicit dynamic analysis procedure performs many small-time increments efficiently. An explicit central-difference time integration rule is used, the operator of which is conditionally stable. The actual stable time increment chosen by ABAQUS/Explicit is often less than $\Delta t_{\text {crit }}$ by a factor of $1 / \sqrt{3}$ in a three-dimensional model. More information about the procedure and numerical implementation can be found in [ㄷ] $]$. As an assessment of the stability, the time step was always found to be below $1.474 \times 10^{-9} \mathrm{~s}$ for all the models corresponding to $l_{e}=1 \mathrm{~mm}$ and $\alpha=0.1$. This critical value can be safe enough for the validity of the results as trailed in [1].

The effect of sample number was also evaluated in [ $[$ ] for the RVEs with the size of $25 \times 25 \times 25$ $\mathrm{mm}$. The variations of the mean value and the standard deviation of stress against the sample number were estimated. Two stress values were considered at the peak and the displacement of $0.03 \mathrm{~mm}$, where the maximum standard deviation was observed. The results indicated that both the mean value and standard deviation of stress tended to be stable after 30 samples. Hence, in this study, 30 random samples per model set are generated and used for simulations and a larger size of RVE $(50 \times 50 \times 50$ $\mathrm{mm}$ ) was selected in order to obtain a better statistical representation.

\section{Simulation results and discussion}

To understand the mechanisms behind the fracture process in concrete and interpret the stress curves in connection with structural features and strain rate, first, the general characteristics of the tensile crack behaviour are recognised. It is achieved using an effective visualisation strategy, which contributes to a qualitative detailed analysis of the morphology of the cracks while initiating and growing. Then, the stress-displacement response and energy dissipation are investigated. In the following, to ease the description of the comparison among the samples, each model is named as "aggregate shape-aggregate volume fraction-strain rate", where the strain rate of 0 is correlated to the static loading condition. Fig. 7 shows a summary of the comparison views followed in the discussion. 


\subsection{Fracture mechanisms}

\subsubsection{Visualisation of fracture process}

The accuracy of a visualisation-based analysis depends on the method and the quality of rendering 3D complex crack network in concrete with such random multiphase structure. It might be impossible to display all the damage details, but it is essential to highlight the dominant crack patterns. The proposed visualisation approach is designed based on two sequential regimes of the damage development and the final fracture that can be mostly observed in each set. The first regime is the micro-crack system and the second one is the mechanism of fracture surface formation. It should be mentioned that here, the final fracture is defined based on the damage status at the end of the simulation. In a physical sense, the term of micro-crack can be interpreted as weak/microdiscontinuity. Such relatively small crack does not always grow sufficiently to contribute to the final fracture while those, which develop enough, generate the fracture surfaces as large discontinuity (or so-called macro-cracks). From the numerical point of view, the micro-cracks can be described by correlating them with the CIEs.

As an example, Fig. 8 is used to better explain the crack mechanisms during loading based on the visualisation methods proposed for all the models. The figure illustrates the model IRG-30-0 loaded in X-direction. To visualise the micro-cracks, the cohesive elements are deleted from the model. It causes some gaps to appear due to the CIEs with low-stiffness and relatively large displacement. This view makes sense of the concept of CZM in modelling cracks in brittle materials. In Fig. 8, to show the micro-cracks, a cross-sectional view perpendicular to the loading direction is used at about midplane $(z=25 \mathrm{~mm})$ while the displacements in X-direction are scaled by 30 for a clearer display. The micro-crack evolution is shown at three displacements up to right before the fracture surface formation. Then, the fracture surfaces are demonstrated in 3D by the CIEs which SDEG values equal to 1 . Like the prior regime, the damage development is displayed in three stages up to when the fracture surface structure is close to its final form. To make the crack visualisation more meaningful, the final fracture state is shown in three ways in the figures. The cross-sectional view enables to recognise which micro-cracks contributed to the final fracture. The first $3 \mathrm{D}$ view indicates the positions of the ultimate fracture morphology against the aggregates. The second 3D view illustrates the exaggerated deformation of the sample in which the CIEs are removed and the displacements in X-direction are scaled by 400 . Moreover, as marked by the dashed line in the first row and shown in 
the last row of Fig. 8, the magnified image is used for more accurate analysis of the crack paths among the aggregates and the voids. The region is selected in the way to include some critical micro-cracks, which eventually evolve to macro-cracks.

\subsubsection{Nucleation and propagation of micro-cracks}

The stress distribution dictates the fracture behaviour of concrete, which itself is affected by the type of loading, the random structural characteristics of the aggregates and their interaction with each other. The local stress is mainly enhanced around the aggregates, where the ITZs exist as the weak bound, and in the small regions between them. Hence, the micro-cracks tend to nucleate and develop along the aggregate surfaces and the narrow paths in the mortar. The potential points, where a crack originates, are called nucleation points. Three geometric properties of the aggregates can significantly influence the formation of these points and the crack growth as described below.

The first is the random location of aggregates with various size/shape. It has the main role in the generation of the non-uniform stress field, which implies the reason behind the random damage event because of the stochastic distribution of the nucleation points. The second is the volume fraction, the effect of which is correlated with the distance among the aggregates. It can be evaluated by tracking the micro-crack paths at $d_{3}$ in the models $\boldsymbol{I R G}$-30-0 (Fig. 8), IRG-35-0 (Fig. 9) and IRG-40-0 (Fig. 10). Generally, the average distance of the aggregates/voids decreases with the increase of their content. The stress filed in the thin tortuous layers of the mortar is drastically enhanced due to the small local surface area. As seen in Figs. 8-11, the cracks tend to pass through the aggregates that are closer to each other and this trend becomes clearer with the increasing aggregate volume fraction. The third is the shape effect, which can be highlighted by a comparison of the micro-crack system between the concrete models $\boldsymbol{I R G - 3 0 - 0 ~ a n d ~} \boldsymbol{S P H}-30-0$, as shown in Figs. 8 and 11. In both models, the distance and the random location of the aggregates would have the same effect, but in IRG-30-0, the shape characteristics (e.g. low-sphericity, low-elongation ratio and low-roundness) cause an additional damage mechanism. The irregular shape of the aggregate surface can intensify the stress concentrations in concrete due to the small local surface area, and the sharp edges and corners found on the aggregate surface. Consequently, in the model IRG-30-0, the number of nucleation points around the aggregates is greater than that in the model $\mathbf{S P H}-30-0$, which suggests that the shape irregularity increases the formation probability of the micro-cracks at more locations and the propagation possibility in different directions. Apart from the aggregate structural characteristics, the 
voids as the initial defects provide easier conditions for the damage development in the mortar. Thus, they can be always found in the way of the critical micro-crack propagation although they have less impact on the fracture process in concrete due to the low volume fraction, i.e. $2 \%$. To further estimate the effect of mesoscopic voids on the mechanical response, a comprehensive parametric study is required (e.g. based on a systematic increase of volume fraction of voids), which is out of the scope of this study but is a subject of ongoing work and will be presented in a future publication.

In the dynamic regime, the micro-crack propagation in concrete is also under the influence of the loading rate. This effect can be recognised by comparing the concrete models IRG-30-0, IRG-30-1, IRG-30-10 and IRG-30-50 shown in Figs. 8 and 12-14, respectively. As the strain rate increases, two rate sensitivity characteristics of the cracks become more distinguishable, including planar propagation direction and a great number of micro-cracks. This behaviour is affected by the inertia forces at the local micro-crack tip level and the energy activation mechanism [66]. The effect is more evident in the magnified selected region presented in the figures. The rate of the micro-crack growth increases in the planar direction and few cracks coalesce with those in the out-of-plane [67]. As a result, under a higher strain rate loading, the damage in concrete is not concentrated at the limited locations like that observed under the static or low-strain rate conditions. In this state, the microcracks are activated from more nucleation points, and the damage development probability increases at more positions. Thus, concrete under a higher strain rate loading endures more damage.

\subsubsection{Formation and final morphology of fracture surface}

The micro-crack system mainly dictates the fracture surface formation and implies the reason behind macro-cracking behaviour of concrete. Henceforth, only, the main characteristics of fracture surface are required to be discussed for each model set. Under static loading, two fracture patterns can be generally detected. In the so-called regular pattern, the macro-cracks evolve and coalesce into one surface structure in which all external edges are located on the RVE boundaries (Figs. 10 and 11). In this state, the specimen is finally broken into two parts. In contrast to this, irregular pattern leads to the formation of two (or probably more) fracture surfaces and breaking of the samples into more than two parts (Figs. 8 and 9). With the increase of aggregate volume fraction, the occurrence possibility of regular pattern raises as the coalescence is dominated by the small distance of aggregates. Among the models IRG-40-0, the regular pattern can be observed in $87 \%$ of the samples while $90 \%$ of the models IRG-30-0 are fractured with an irregular mode. These probability values seem to justify why 


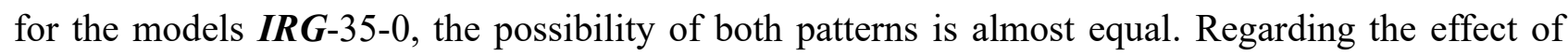
aggregate shape, it can be found that the spherical aggregates induce regular pattern according to the fracture morphology of $93 \%$ of the models $\boldsymbol{S P H}-30-0$. Thus, such simplified model cannot accurately predict the fracture pattern because it disregards the effect of shape irregularity on the cracking process as discussed earlier.

Under dynamic loading, the damage evolution in concrete can be generally described by the formation of the quasi-parallel fracture surfaces and the planar crack development at the same time. In the regimes of the low-moderate strain rate, e.g. the models IRG-30-1 and IRG-30-10 (Figs. 12 and 13), the pattern is almost similar to that under the static loading (Fig. 8) with only few differences, which are affected by the change in the micro-crack coalescence behaviour. Under high strain rate loading, the number of the fracture surfaces increases during the damage development, as illustrated in Fig. 14 in two view angles, which indicates that more micro-cracks could trigger the macro-cracks. The simulated micro- and macro-crack patterns of concrete here are generally consistent with those observed in the experiments [67], which were conducted under static $(0.0001 \mathrm{GPa} / \mathrm{s})$, moderate $(39$ $\mathrm{GPa} / \mathrm{s})$ and high $(1685 \mathrm{GPa} / \mathrm{s})$ loading rate regimes. As seen in Fig. 15 and described in [67], the cracks were mainly formed at the interfaces of the irregular aggregates in concrete and passed through the small distances among aggregates. The crack propagation directions changed with the increase of loading rate as the ways explained earlier for the models.

In a view of quantitative analysis of crack morphology, the number of CIEs created the fracture surface is counted at the locations, where most of the macro-cracks have already been formed for all the models. The number of failed elements can represent the macro-damage level induced by different loading conditions. A comparison of the models provides an interpretation of the damage levels in relation to the fracture patterns. To show the general trend, the graphical representation of the data in Fig. 16 illustrates the number of failed CIE elements for each model of concrete. The data are connected based on the comparison views presented in Fig. 7. As seen, the number of elements is higher for the cases of regular pattern, which implies that the fracture surfaces are developed more abundantly, and the macro-cracks become more evident/detectable. In addition, the strain rate has a more significant influence than the structural factors on the tensile fracture of concrete. Here, the macro-damage level was approximated based on the non-uniform mesh, which could be disputable 
for analysing such random models. In the next section, the damage level is extended in relation to the dissipated energy as a more reliable variable for both micro- and macro-crack systems.

\subsection{Stress and energy dissipation responses}

So far, the tensile fracture of concrete has been analysed based on the visualisation of cracks. In the following, first, the main influences of damage mechanisms on the stress response and the energy dissipation response are investigated in reference to the stress-displacement curves of concrete with various aggregate volume fractions and shapes under static and dynamic tension. Then, the critical values of stress and dissipated energy identified on the curves are compared among all the models.

Before discussing the results, the method of plotting data needs to be described. For each sample, the stress is calculated as the sum of the nodal reaction forces on the loaded face divided by its initial area, i.e. $50 \times 50 \mathrm{~mm}^{2}$. Then, the representative curve is obtained by averaging the data during loading for all models per set. To ease the comparison, the stress and the dissipated energy values are normalised to the mortar tensile strength $(4 \mathrm{MPa})$ and fracture energy $(0.06 \mathrm{~N} / \mathrm{mm})$ multiplied by the cross-sectional area, respectively, as dimensionless parameters. The corresponding standard deviation of the data is also presented. Like the normalised stress-displacement curves, the representative mean values of the normalised dissipated energy and the associated standard deviation are plotted versus the displacement for each model set. According to the comparison strategy proposed (Fig. 7), the corresponding curves are displayed in the same figure.

\subsubsection{Stress-displacement curve}

Fig. 17 shows the normalised stress against displacement for the concrete models $\boldsymbol{I R G}$-30-0 and $\boldsymbol{S P H}$ 30-0. For comparison, the experimental results of tensile stress-displacement response of concrete under static loading reported in [68] are also plotted. It can be observed that the simulation results are generally consistent with the experiments under static loading in terms of stress values at the characteristic points, e.g. the peak stress $S^{\operatorname{Max}}$ (or the concrete tensile strength) at the peak point (also called micro-point) and the critical stress $S^{C r}$ at the macro-point corresponding to the maximum standard deviation. In Fig. 17, the characteristic points and the approximated range corresponding to macro-crack phase is graphically indicated by the dashed-dot lines and the coloured areas, respectively, as guides to the eyes. The discrepancies between simulation results and experimental data can be attributed to the differences in sample preparation, experimental testing condition and concrete features (e.g. void or aggregate content), etc. It is worth mentioning that this 
study does not aim to generalise the findings but to achieve insight and suggest hypothesis and directions for concrete design. Regarding the aggregate shape effect, there is no considerable difference between the models IRG-30-0 and $\boldsymbol{S P H}-30-0$. The value of $S^{M a x}$ at the displacement of $0.011 \mathrm{~mm}$ is about 0.87 for both models with a discrepancy of only $2 \%$, while the standard deviations are in the range between 0.015 and 0.007 . It can be noted that the uniaxial tensile strength of concrete does not significantly change with the shape of aggregate in view of stress-displacement response. For the models IRG-30-0, the various micro-cracking scenarios lead to a higher standard deviation and about after the macro-point $(d>0.031 \mathrm{~mm})$ the stress level is higher due to the irregular fracture pattern. In this case, $S^{C r}$ is $29 \%$ greater compared to the model $\boldsymbol{S P H}-30-0$. Unlike the shape, the aggregate volume fraction has more evident effects. As seen in Fig. 18, the curve related to the model with less aggregate content is above the other ones and the micro-point shifts to the left on the displacement axis from 0.011 to $0.009 \mathrm{~mm}$ with the increase of aggregate volume fraction from $30 \%$ to $40 \%$. On the graph, the curve tendency regarding the volume fraction increment is schematically demonstrated by the arrow. Such change in stress-displacement response can be correlated to increased brittleness of concrete. The value of $S^{\operatorname{Max}}$ for the model IRG-30-0 decreases by about 12\% to 0.77 and 0.76 respectively for the models $I R G-35-0$ and $I R G-40-0$, whilst $S^{C r}$ reduces by around $28 \%$ from 0.303 to 0.22 and 0.21 , respectively. Similar to the experiments [52], the simulation results also prove that $30 \%$ of coarse aggregate is the optimum volume fraction to achieve the maximum strength for the plain concrete under uniaxial static tension.

Regarding dynamic loading, the normalised stress-displacement responses with respect to the strain rates exhibit distinct changes in different phases of the fracture process in concrete, as seen in Fig. 19a. In the elastic region before the micro-point, the stiffness of concrete raises significantly with the change of loading condition from the static loading to the dynamic loading with low-moderate strain rates and then to high strain rate. To quantitatively demonstrate this effect, the representative stiffness value can be linearly estimated by dividing the reaction force by displacement at micro-point. After calculations, it can be found that the increment percentages of stiffness with respect to strain rate are $55 \%$ (from 0 to $1 \mathrm{~s}^{-1}$ ), $2 \%$ (from 1 to $10 \mathrm{~s}^{-1}$ ) and $27 \%$ (from 10 to $50 \mathrm{~s}^{-1}$ ). The stiffness variation can be associated with the change of material strength. For the static and low-moderate loads, $S^{\text {Max }}$ is almost equal (0.877-0.920), whereas it increases to 1.012 for the model IRG-30-50, the corresponding strain rate of which is $50 \mathrm{~s}^{-1}$. When the static regime changes to the dynamic one with 
a low strain rate of $1 \mathrm{~s}^{-1}$, the fracture surface formation initiates earlier, at $d=0.023 \mathrm{~mm}$. A comparison of the crack evolution in the models $\boldsymbol{I R G}-30-0$ and $\boldsymbol{I R G}-30-1$ (Figs. 8 and 12) indicates that the crack patterns in them are similar during the fracture process except for some few macrocracks. Thus, it can be interpreted that the inertia force may accelerate crack growth and results in an earlier fracture. Moreover, the micro-crack density raises with the increase of strain rate, which allows the sample to be deformed more easily. Hence, the displacement value at the macro-point increases to 0.043 and $0.096 \mathrm{~mm}$ for the models $\boldsymbol{I R G}-30-10$ and $\boldsymbol{I R} \boldsymbol{G}-30-50$, while no specific trend can be found with $S^{C r}$. This changing pattern of the stress-displacement curve is consistent with those found in the experiments [67]. For comparison, the experimental results of stress-displacement response of concrete under static and dynamic tension are shown in Fig. 19b. Among three cases reported for the high strain rate loading regimes, the curve with a very wide peak has the most similarity to the curve shown for the model IRG-30-50 (Fig. 19a). For this case, more than one macro-crack was formed before the specimen was completely broken like what observed in the simulations, e.g. Fig. 14. As no strain-rate terms were incorporated into the constitutive material properties of different phases of concrete, the dynamic strength enhancement simulated can only be attributed to the meso-structural effects of concrete. Thus, the stress levels between simulations and experiments are not comparable.

\subsubsection{Dissipated energy}

The dissipated energy and the corresponding standard deviation as a function of displacement are also displayed in Figs. 17-19. The changes of dissipated energy-displacement curves are generally like those observed for the stress-displacement response and related to the cracking system. The dissipated energy-displacement curves do not differ significantly in Fig. 17 whereas, in Fig. 18, they deviate more and more from each other toward fracture surface formation and final fracture. The most differences are observed when the strain rate changes (Fig. 19).

The amount of energy, $E^{\text {Micro }}$, at macro-point equals to the energy dissipated during micro-crack evolution. The lost energy through the fracture surface formation, $E^{\text {Macro }}$, can be estimated by subtracting $E^{\text {Micro }}$ from the total energy $E^{\text {Tot }}$ at the end of loading as the slope of the curve becomes almost flat, although in some cases, especially those with irregular fracture pattern, $E^{\text {Macro }}$ might be underestimated since if the simulation continued, the sample would remain under tension till it was mostly fragmented into some pieces. It can be clearly seen that $E^{\text {Micro }}$ is always significantly greater than $E^{\text {Macro }}$, which implies that the micro-crack system contributes more to 
concrete failure. In Section 4.1.3, the damage level was evaluated based on the number of failed elements. Here, the level of damage is estimated based on the total energy dissipated by the fracture process. More specifically, the data analysis about the values of $E^{\text {Micro }}$ and $E^{\text {Macro }}$ provides a physical sense to understand how much each damage mechanism contributes to the final fracture of concrete. To do so, the dissipated energy-displacement curves are interpreted with the aid of Fig. 20, in which the same data are shown in two different ways. A stacked column graph constructed by the variables $E^{\text {Micro }}$ and $E^{\text {Macro }}$ is employed to demonstrate $E^{\text {Tot }}$ for each model set, while the relative percentages of these variables with respect to the total energy are depicted using a $100 \%$ stacked column chart. It can be observed that the contribution of micro-cracks to the fracture of concrete is above $75 \%$, which indicates the importance of this phase in the analysis of damage evolution in concrete in the view of energy dissipation.

For models IRG-30-0 and $\boldsymbol{S P H}-30-0$, the values of $E^{T o t}$ are $\sim 1.2$, but the $E^{\text {Macro }}$ value is greater for the model with the realistic shape aggregates, i.e. IRG-30-0 (0.31), which suggests that the irregular fracture pattern results in more intense macro-damage. In general, for the models failed with a regular pattern, the contribution of macro-cracks is about $20 \%$, while for the irregular-case it is slightly higher, equal to $24 \%$. As the volume fraction of aggregates increases from 30 to $40 \%, E^{T o t}$ decreases by $14 \%$ to 1.104 , which can be attributed to the increased brittleness. In addition, $E^{\text {Tot }}$ raises significantly with the increase of strain rate. With respect to the static case, the increment factors are $1.2,2.55$ and 6.4 for the strain rates of 1,10 and $50 \mathrm{~s}^{-1}$, respectively, which just reflect the mesoscopic structural effects on the dissipation energy. Compared to IRG-30-0 under static loading, $E^{\text {Macro }}$ of IRG-30-1 under dynamic loading is decreased by $23 \%$, in which the macro-cracks grow faster and reach sooner to the final fracture and thus, the macro-damage phase has less contribution during the fracture process compared to the static case (15\% versus $24 \%)$. However, the formation of more fracture surfaces under higher strain rates leads to an increase of $E^{\text {Macro }}$ and its contribution percentage to the tensile fracture of concrete. In summary, the influence of strain rates on the mechanical response of concrete under uniaxial tensile loading is more significant than the structural effect in terms of stress and dissipated energy.

\section{Conclusions}

In this study, a computational framework for investigating the effects of structural features and strain rate on uniaxial tensile fracture of concrete was developed. A novel four-phase mesostructure model 
of concrete consisting of coarse aggregates, mortar, interfacial transition zone (ITZ) between them and voids with good controllability of structural features like size distribution and easy-measurability of shape distribution was employed. The generated mesostructure of concrete with real characteristics of aggregate shape and size distribution was then incorporated into the finite element cohesive zone model for simulating the fracture process in concrete. Afterwards, the effects of aggregate shape, aggregate content and loading rate on fracture process in concrete were estimated and discussed in detail. Based on the simulation results, the main conclusions can be drawn as follows:

- The tensile fracture process in concrete was composed of two stages. The first was the microcrack phase, which might not be detectable easily in the experiments. The simulation results showed that the micro-crack phase played a dominant role in the damage evolution in concrete with over $75 \%$ contribution to the energy dissipation mechanism. The second was the fracture surface formation phase in which the macro-cracks were relatively more visible to the naked eyes in practice.

- Two main structural factors stimulated the micro-cracks initiation and growth in concrete that would significantly enhance local stress concentrations: (1) The small distance between the aggregates, and (2) the irregular shape characteristics of aggregates such as the small local surface area and high angularity. The effect of the first factor became more evident with the increase of aggregate volume fraction and the model with spherical aggregates was not able to simulate the influence of the second factor due to their perfectly round shape. Moreover, in the static regime, it was observed that if the first factor was dominant, the occurrence possibility of the regular pattern (one fracture surface) would increase, otherwise the sample would be likely fractured with the irregular pattern (multiple fracture surfaces). As the strain rate increased, more cracks were formed in the quasi-parallel planes perpendicular to the load direction.

- In general, the structural properties of aggregates did not significantly affect the stress- and dissipated energy-displacement curves except the reduction of uniaxial tensile strength and the dissipated energy with the increase of aggregate volume fraction from $30 \%$ to $40 \%$. In contrast, with the increase of strain rate, concrete became stiffer in the elastic region and the tensile strength and total dissipated energy of concrete were significantly increased.

- It should be noted that the stress or dissipation energy variations do not clearly reflect the crack behaviour affected by the structural effect, e.g. aggregate shape. Therefore, it is essential to 
simultaneously consider stress, dissipated energy and crack morphology for a comprehensive analysis of mechanical performance of concrete, as the crack initiation and growth not only affect the sensitivity of tensile fracture of concrete but also influence the durability and service life of concrete structures.

It is worth mentioning that in a relevant study to the current one, the effect of aggregate shape on the uniaxial compressive fracture behaviour of concrete was investigated using the concrete damage plasticity (CDP) method [69]. It can be considered as complementary to this work. The CDP approach is more accurate in compression mode compared to the CZM presented here. Moreover, in a view of analysis of crack morphology, the CDP model better represents the crushing process using the obtained damage-cloud.

Future study will focus on the simulations of dynamic increase factor of concrete under both tension and compression modes. In order to achieve this, two modifications will be performed for the sake of accuracy and efficiency. First, the developed model will be improved at material level based on the specific type of concrete and experimental validation. Second, the large number of cohesive elements will be reduced using different damage models for the mortar in concrete to reduce the computational cost. These are the subjects of ongoing research, the results of which will be presented in future publications.

\section{Acknowledgements}

The authors gratefully acknowledge the financial support from the Engineering and Physical Sciences Research Council (EPSRC), UK under Grant No. EP/R041504/1 and the Royal Society, UK under Award No. IEC\NSFC $\backslash 191417$. The research was also supported by Visiting Researcher Fund Program of State Key Laboratory of Water Resources and Hydropower Engineering Science, China under Award No. 2019SGG01.

\section{References}

[1] O. Y1lmaz, J.-F. Molinari, A mesoscale fracture model for concrete, Cement and Concrete Research 97 (2017) 84-94.

[2] A. Sarja, E. Vesikari, Durability design of concrete structures, CRC Press2004.

[3] M.N. Hassoun, A. Al-Manaseer, Structural concrete: theory and design, John wiley \& sons2020.

[4] R.I. Gilbert, G. Ranzi, Time-dependent behaviour of concrete structures, CRC Press2010. 
[5] H. Reinhardt, J. Weerheijm, Tensile fracture of concrete at high loading rates taking account of inertia and crack velocity effects, International Journal of Fracture 51(1) (1991) 31-42.

[6] D. Shi, X. Chen, Flexural tensile fracture behavior of pervious concrete under static preloading, Journal of Materials in Civil Engineering 30(11) (2018) 06018015.

[7] S.A. Paschalis, A. Lampropoulos, Ultra high performance fiber reinforced concrete under cyclic loading, ACI Materials 113(4) (2016) 419-427.

[8] X. Wang, M. Zhang, A.P. Jivkov, Computational technology for analysis of 3D meso-structure effects on damage and failure of concrete, Int J Solids Struct 80 (2016) 310-333.

[9] S. Bhowmik, S. Ray, An experimental approach for characterization of fracture process zone in concrete, Engineering Fracture Mechanics 211 (2019) 401-419.

[10] M. Ghasemi, M.R. Ghasemi, S.R. Mousavi, Investigating the effects of maximum aggregate size on self-compacting steel fiber reinforced concrete fracture parameters, Constr Build Mater 162 (2018) 674-682.

[11] V. Afroughsabet, T. Ozbakkaloglu, Mechanical and durability properties of high-strength concrete containing steel and polypropylene fibers, Constr Build Mater 94 (2015) 73-82.

[12] J.F. Unger, S. Eckardt, C. Könke, Modelling of cohesive crack growth in concrete structures with the extended finite element method, Computer Methods in Applied Mechanics and Engineering 196(41) (2007) 4087-4100.

[13] Y. Wang, X. Li, Y. Wu, C. Lin, B. Zhang, Experimental study on meso-damage cracking characteristics of RSA by CT test, Environmental Earth Sciences 73(9) (2015) 5545-5558.

[14] S.D. Abyaneh, H.S. Wong, N.R. Buenfeld, Computational investigation of capillary absorption in concrete using a three-dimensional mesoscale approach, Comp Mater Sci 87 (2014) 54-64.

[15] L. Tang, W. Zhou, X. Liu, G. Ma, M. Chen, Three-dimensional mesoscopic simulation of the dynamic tensile fracture of concrete, Engineering Fracture Mechanics 211 (2019) 269-281.

[16] P.E. Petersson, Fracture energy of concrete: Practical performance and experimental results, Cement and Concrete Research 10(1) (1980) 91-101.

[17] F. Bencardino, L. Rizzuti, G. Spadea, R.N. Swamy, Implications of test methodology on postcracking and fracture behaviour of Steel Fibre Reinforced Concrete, Composites Part B: Engineering 46 (2013) 31-38. 
[18] A. Gangnant, J. Saliba, C. La Borderie, S. Morel, Modeling of the quasibrittle fracture of concrete at meso-scale: Effect of classes of aggregates on global and local behavior, Cement Concrete Res 89 (2016) 35-44.

[19] R.R. Pedersen, A. Simone, L.J. Sluys, Mesoscopic modeling and simulation of the dynamic tensile behavior of concrete, Cement and Concrete Research 50 (2013) 74-87.

[20] M.F. Benedetto, A. Caggiano, G. Etse, Virtual elements and zero thickness interface-based approach for fracture analysis of heterogeneous materials, Computer Methods in Applied Mechanics and Engineering 338 (2018) 41-67.

[21] L. Snozzi, F. Gatuingt, J.F. Molinari, A meso-mechanical model for concrete under dynamic tensile and compressive loading, International Journal of Fracture 178(1) (2012) 179-194.

[22] P. Thilakarathna, K.K. Baduge, P. Mendis, V. Vimonsatit, H. Lee, Mesoscale modelling of concrete - A review of geometry generation, placing algorithms, constitutive relations and applications, Engineering Fracture Mechanics 231 (2020) 106974.

[23] H. Ma, W. Xu, Y. Li, Random aggregate model for mesoscopic structures and mechanical analysis of fully-graded concrete, Computers \& Structures 177 (2016) 103-113.

[24] K. Janardhana Reddy, A. Das, Shape characterisation of aggregates in three dimension, European Journal of Environmental and Civil Engineering (2019) 1-15.

[25] A. Amirjanov, K. Sobolev, Optimization of a computer simulation model for packing of concrete aggregates, Particulate Science and Technology 26(4) (2008) 380-395.

[26] X.-Q. Zhou, Y. Xia, Mesoscale modeling of concrete under dynamic split tension, Journal of Earthquake and Tsunami 7(03) (2013) 1350028.

[27] P. Thilakarathna, K.K. Baduge, P. Mendis, V. Vimonsatit, H. Lee, Mesoscale modelling of concrete-a review of geometry generation, placing algorithms, constitutive relations and applications, Engineering Fracture Mechanics (2020) 106974.

[28] S. Naderi, M. Zhang, An integrated framework for modelling virtual 3D irregulate particulate mesostructure, Powder Technology 355 (2019) 808-819.

[29] Y. Huang, D. Yan, Z. Yang, G. Liu, 2D and 3D homogenization and fracture analysis of concrete based on in-situ X-ray Computed Tomography images and Monte Carlo simulations, Engineering Fracture Mechanics 163 (2016) 37-54. 
[30] Y. Liu, F. Gong, Z. You, H. Wang, Aggregate morphological characterization with 3D optical scanner versus X-Ray computed tomography, Journal of Materials in Civil Engineering 30(1) (2018) 04017248.

[31] P. Carrara, R. Kruse, D.P. Bentz, M. Lunardelli, T. Leusmann, P. Varady, L. De Lorenzis, Improved mesoscale segmentation of concrete from 3D X-ray images using contrast enhancers, Cement and Concrete Composites 93 (2018) 30-42.

[32] Z. Qian, Multiscale modeling of fracture processes in cementitious materials, (2012).

[33] Z. Gyurkó, R. Nemes, Fracture modelling of normal concrete using different types of aggregates, Engineering Failure Analysis 101 (2019) 464-472.

[34] R. Zhou, Z. Song, Y. Lu, 3D mesoscale finite element modelling of concrete, Computers \& Structures 192 (2017) 96-113.

[35] J. Zhang, Z. Wang, H. Yang, Z. Wang, X. Shu, 3D meso-scale modeling of reinforcement concrete with high volume fraction of randomly distributed aggregates, Constr Build Mater 164 (2018) $350-361$.

[36] M.E. Kutay, H.I. Ozturk, A.R. Abbas, C. Hu, Comparison of 2D and 3D image-based aggregate morphological indices, International Journal of Pavement Engineering 12(4) (2011) 421-431.

[37] H. Zhang, P. Sheng, J. Zhang, Z. Ji, Realistic 3D modeling of concrete composites with randomly distributed aggregates by using aggregate expansion method, Constr Build Mater 225 (2019) 927940.

[38] R. Zhou, Y. Lu, A mesoscale interface approach to modelling fractures in concrete for material investigation, Constr Build Mater 165 (2018) 608-620.

[39] Y.K. Hwang, J.E. Bolander, Y.M. Lim, Simulation of concrete tensile failure under high loading rates using three-dimensional irregular lattice models, Mechanics of Materials 101 (2016) 136-146. [40] K. Mikeš, F. Bormann, O. Rokoš, R.H. Peerlings, Modelling of crack propagation: Comparison of discrete lattice system and cohesive zone model, Acta Polytechnica CTU Proceedings 26 (2020) $39-44$.

[41] X. Wang, A.P. Jivkov, Combined numerical-statistical analyses of damage and failure of 2D and 3D mesoscale heterogeneous concrete, Mathematical Problems in Engineering 2015 (2015).

[42] Y. Lu, Z. Tu, Mesoscale modelling of concrete for static and dynamic response analysis-Part 2: numerical investigations, Structural Engineering and Mechanics 37(2) (2011) 215-231. 
[43] Y.H. Zhang, Q.Q. Chen, Z.Y. Wang, J. Zhang, Z.H. Wang, Z.Q. Li, 3D mesoscale fracture analysis of concrete under complex loading, Engineering Fracture Mechanics 220 (2019).

[44] O. Yilmaz, J.F. Molinari, A mesoscale fracture model for concrete, Cement and Concrete Research 97 (2017) 84-94.

[45] N. Benkemoun, P. Poullain, H. Al Khazraji, M. Choinska, A. Khelidj, Meso-scale investigation of failure in the tensile splitting test: Size effect and fracture energy analysis, Engineering fracture mechanics 168 (2016) 242-259.

[46] G. Chen, Y.F. Hao, H. Hao, 3D meso-scale modelling of concrete material in spall tests, Mater Struct 48(6) (2015) 1887-1899.

[47] J. Hu, P. Stroeven, Shape characterisation of particles in concrete technology, Proceedings of the 6th International Symposium on Cement \& Concrete and CANMET/ACI International Symposium on Concrete Technology for Sustainable Development, Vols 1 and 2 (2006) 929-937.

[48] J.W. Bullard, E.J. Garboczi, Defining shape measures for 3D star-shaped particles: Sphericity, roundness, and dimensions, Powder Technology 249 (2013) 241-252.

[49] T.M. Al Rousan, Characterization of aggregate shape properties using a computer automated system, Texas A\&M University, 2005.

[50] C. Mora, A. Kwan, Sphericity, shape factor, and convexity measurement of coarse aggregate for concrete using digital image processing, Cement and concrete research 30(3) (2000) 351-358.

[51] A. Kwan, C. Mora, Effects of various shape parameters on packing of aggregate particles, Magazine of concrete Research 53(2) (2001) 91-100.

[52] T.J. Hirsch, Modulus of elasticity iof concrete affected by elastic moduli of cement paste matrix and aggregate, Journal Proceedings, 1962, pp. 427-452.

[53] O.K. Hunter, Characterization of pore structure and crack propagation in concrete using X-Ray computed tomography, 2004.

[54] J.M. Wang, A.P. Jivkov, D.L. Engelberg, Q.M. Li, Meso-scale modelling of mechanical behaviour and damage evolution in normal strength concrete, Procedia Struct Inte 13 (2018) 560-565. [55] Z. Wu, W. Cui, L. Fan, Q. Liu, Mesomechanism of the dynamic tensile fracture and fragmentation behaviour of concrete with heterogeneous mesostructure, Constr Build Mater 217 (2019) 573-591. 
[56] A. Tabiei, W. Zhang, Cohesive element approach for dynamic crack propagation: Artificial compliance and mesh dependency, Engineering Fracture Mechanics 180 (2017) 23-42.

[57] N. Blal, L. Daridon, Y. Monerie, S. Pagano, Criteria on the artificial compliance inherent to the intrinsic cohesive zone, Comptes Rendus Mécanique 339(12) (2011) 789-795.

[58] Y. Huang, S. Hu, Z. Gu, Y. Sun, Fracture behavior and energy analysis of 3D concrete mesostructure under uniaxial compression, Materials 12(12) (2019) 1929.

[59] W. Trawiński, J. Bobiński, J. Tejchman, Two-dimensional simulations of concrete fracture at aggregate level with cohesive elements based on X-ray $\mu \mathrm{CT}$ images, Engineering Fracture Mechanics 168 (2016) 204-226.

[60] Y.J. Huang, Z.J. Yang, X.W. Chen, G.H. Liu, Monte Carlo simulations of meso-scale dynamic compressive behavior of concrete based on X-ray computed tomography images, International Journal of Impact Engineering 97 (2016) 102-115.

[61] Z. Song, Y. Lu, Mesoscopic analysis of concrete under excessively high strain rate compression and implications on interpretation of test data, International Journal of Impact Engineering 46 (2012) 41-55.

[62] L.J. Malvar, C.A. Ross, Review of strain rate effects for concrete in tension, ACI Materials Journal 95 (1998) 735-739.

[63] S. Gao, Nonlinear finite element failure analysis of bolted steel-concrete composite frame under column-loss, J Constr Steel Res 155 (2019) 62-76.

[64] O. Miller, L. Freund, A. Needleman, Modeling and simulation of dynamic fragmentation in brittle materials, International Journal of Fracture 96(2) (1999) 101-125.

[65] ABAQUS: User's Manual, Version 6.12, Dassault Systèmes Simulia Corp, United States, 2012.

[66] J. Ožbolt, K.K. Rah, D. Meštrović, Influence of loading rate on concrete cone failure, International Journal of Fracture 139(2) (2006) 239-252.

[67] I. Vegt, V. Breugel, J. Weerheijm, Failure mechanisms of concrete under impact loading, Fracture Mechanics of Concrete and Concrete Structures, FraMCoS-6 1 (2007) 579-587.

[68] D.A. Hordijk, Tensile and tensile fatigue behaviour of concrete; experiments, modelling and analyses, Heron 37(1) (1992).

[69] S. Naderi, M. Zhang, Meso-scale modelling of compressive fracture in concrete with irregularly shaped aggregates, Cement Concr. Res. 140 (2021) 106317. 
Graphical Abstract

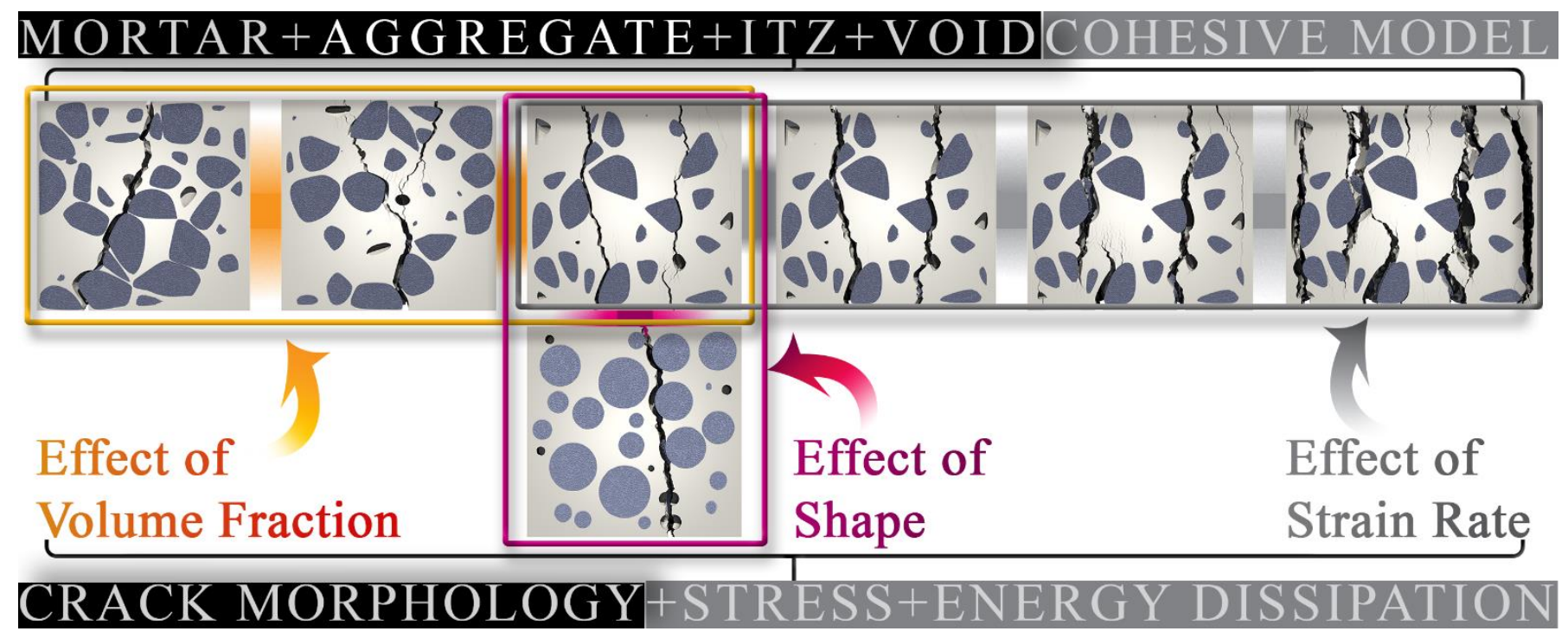




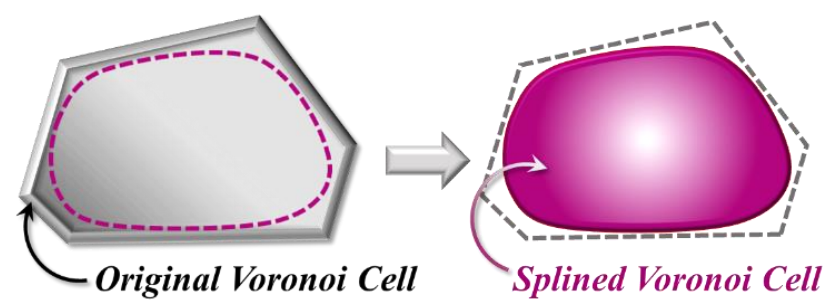

Fig. 1. Schematic of the transformation of the original Voronoi cells by the splining process.

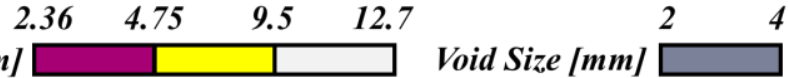 Agg. Size [mm] Small Medium Large}

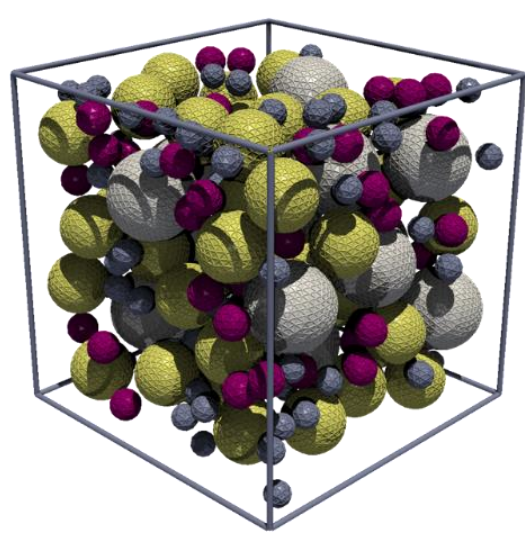

(a) Model SPH

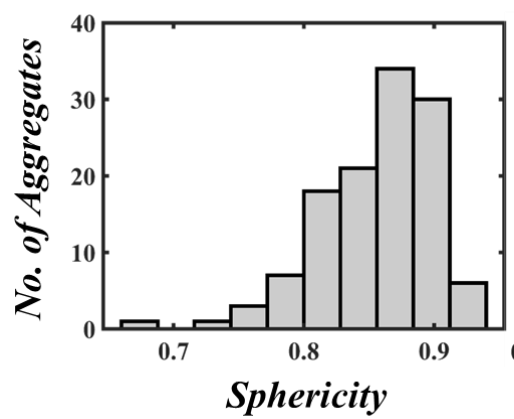

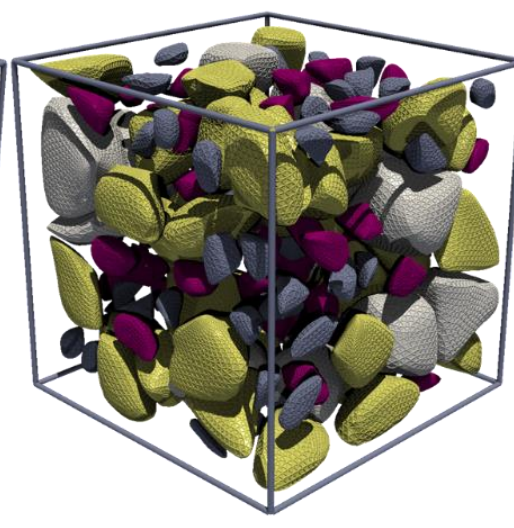

(b) Model IRG

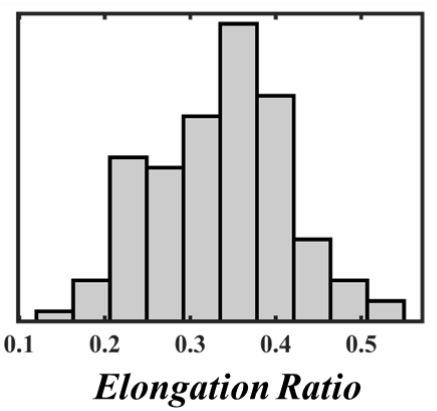

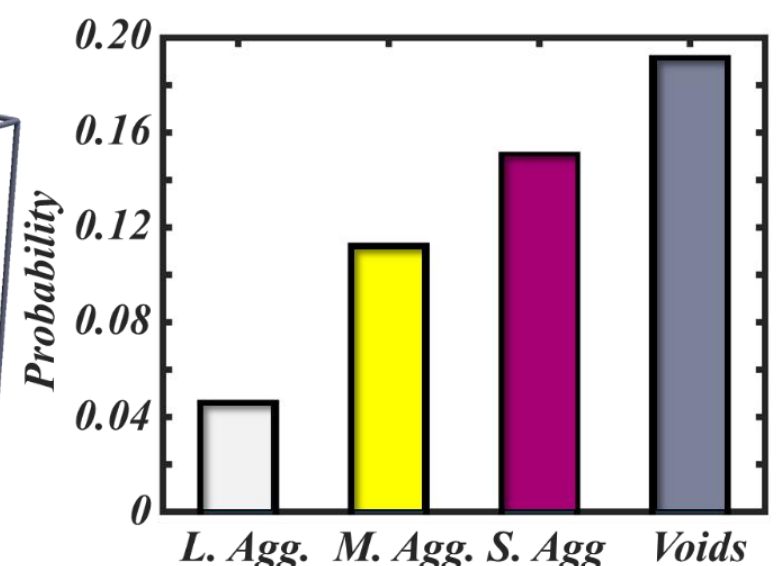

(c)

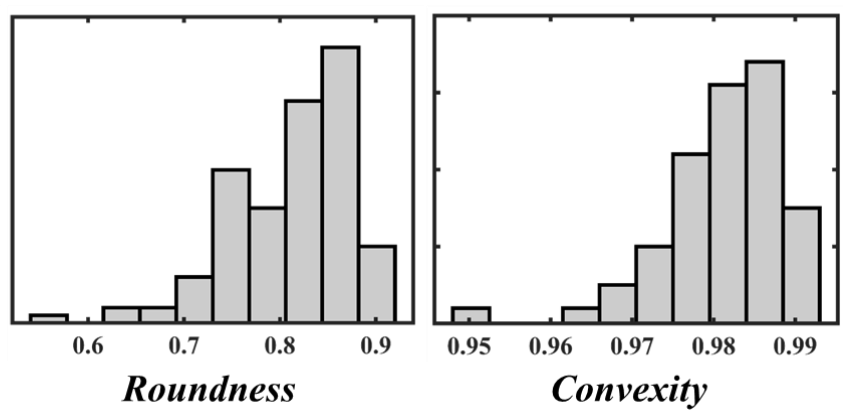

(d)

Fig. 2. Mesostructure models of concrete with spherical (a) and irregular (b) aggregates and $2 \%$ voids. The models are coloured according to the size distribution, which is typically obtained after the size configuration based on Fuller's grading curve. For the model IRG with 30\% aggregates, the representative probability size distribution is plotted against the size segmentations. Also, the example of shape distribution (d) is presented with respect to different shape parameters. 

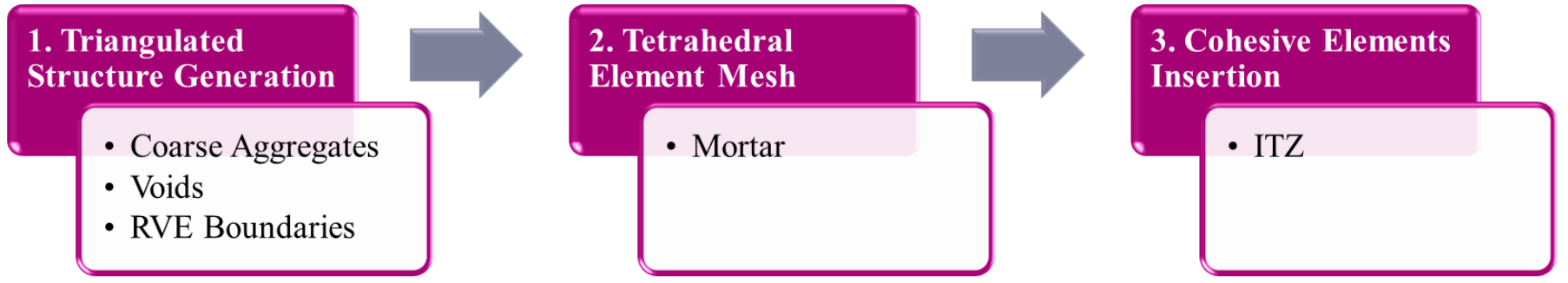

Fig. 3. Main steps to generate the 3D concrete mesostructure along with the phases formed in each stage.

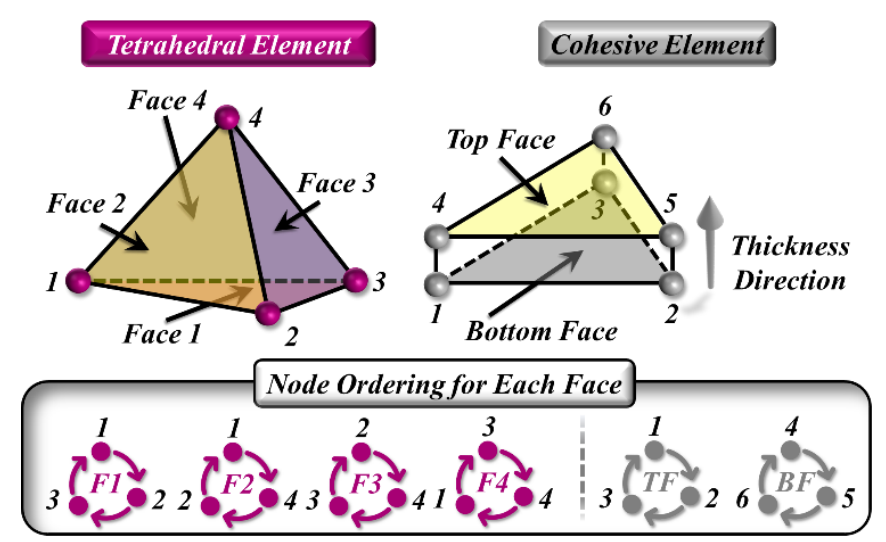

(a)

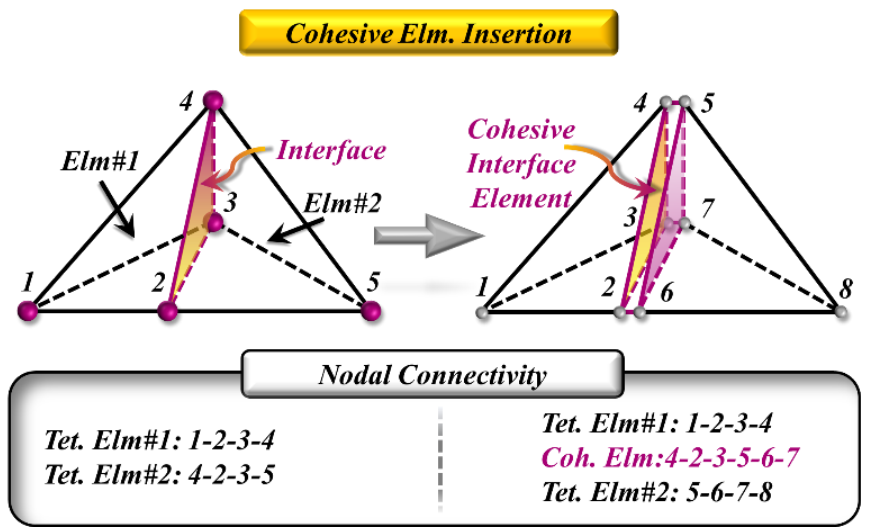

(b)

Fig. 4. (a) Node ordering and face numbering for tetrahedral and cohesive elements. (b) Inserting a cohesive element at the interface of two tetrahedral elements. 


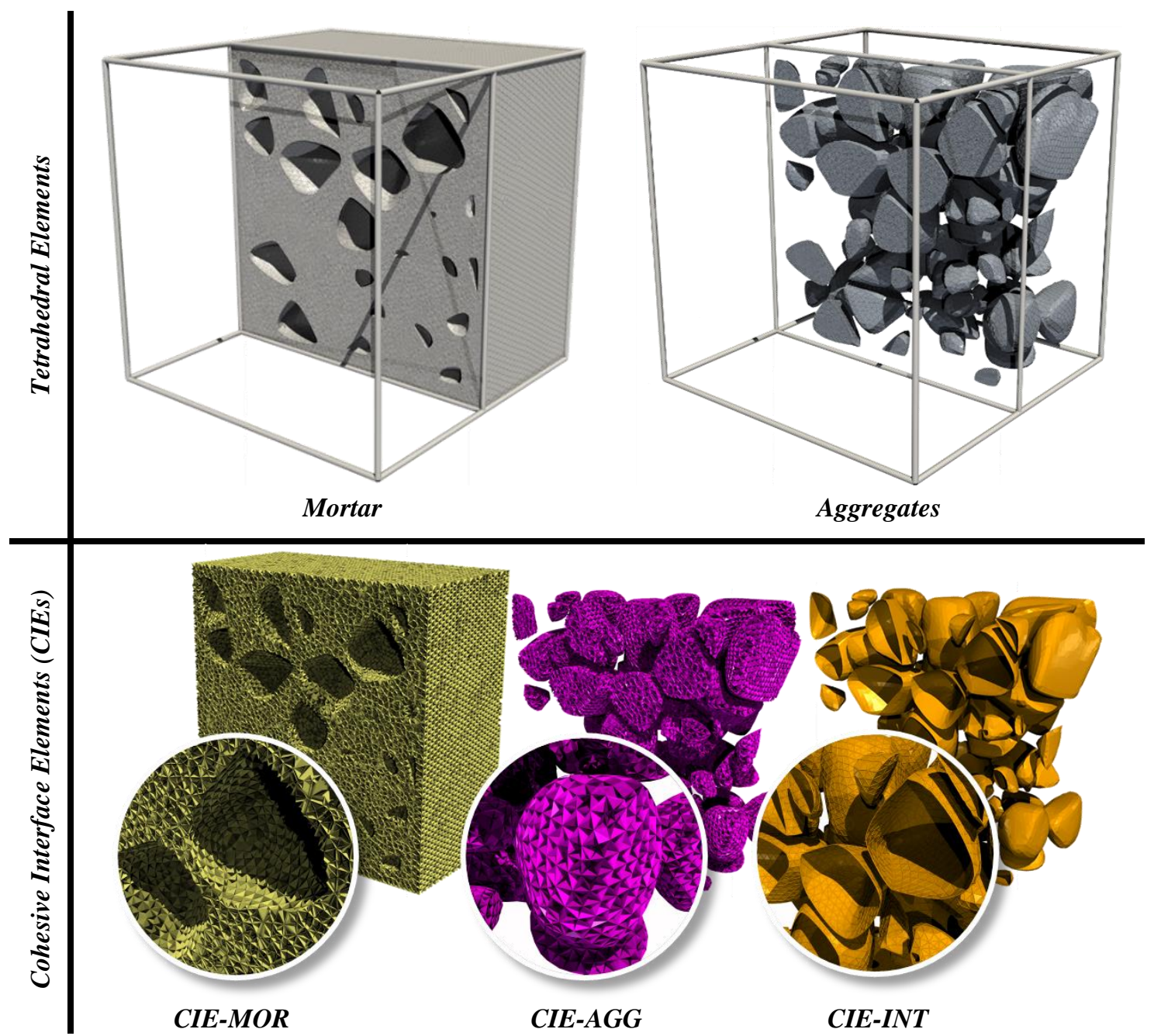

Fig. 5. Different element sets displayed with respect to the element type and the material IDs, in the cross-sectional views for the model $I R \boldsymbol{G}$ with $30 \%$ aggregates.

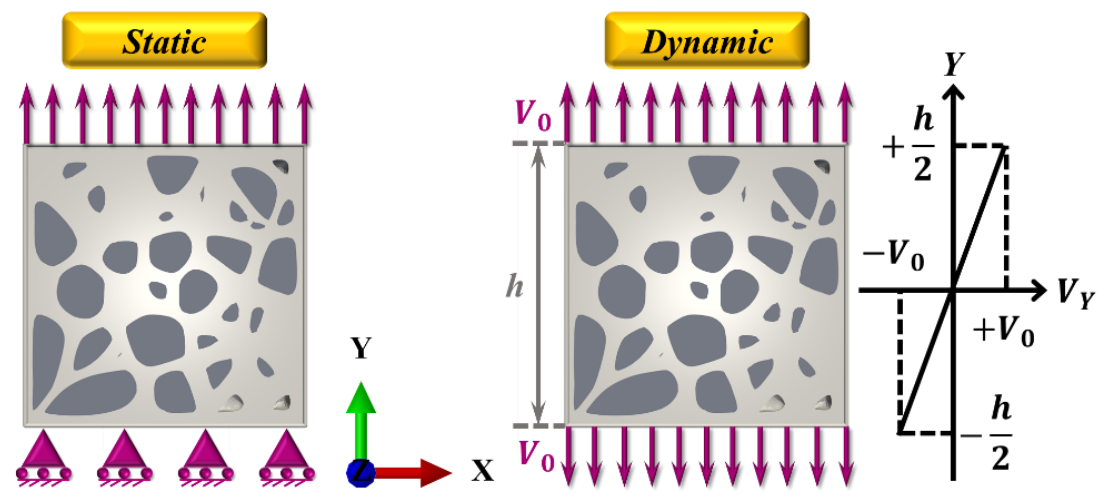

Fig. 6. Schematic diagrams of the uniaxial tensile loading applied on the RVE under static and dynamic conditions. 


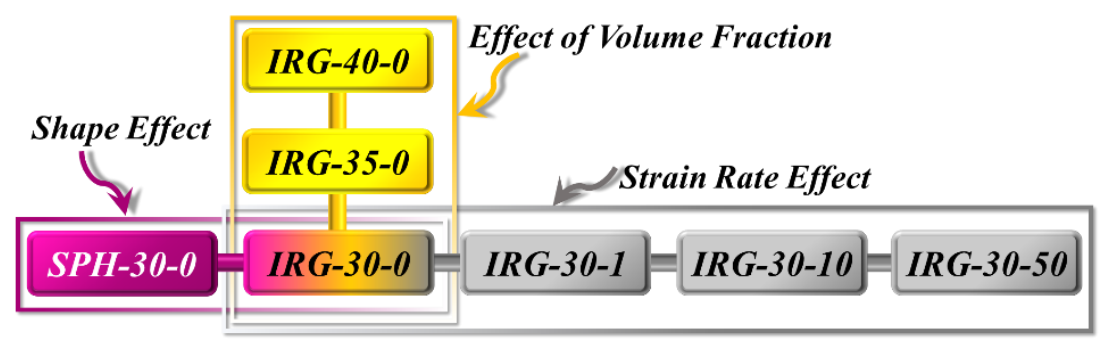

Fig. 7. Comparison views to analyse the numerical results with respect the structural parameters and the strain rate.
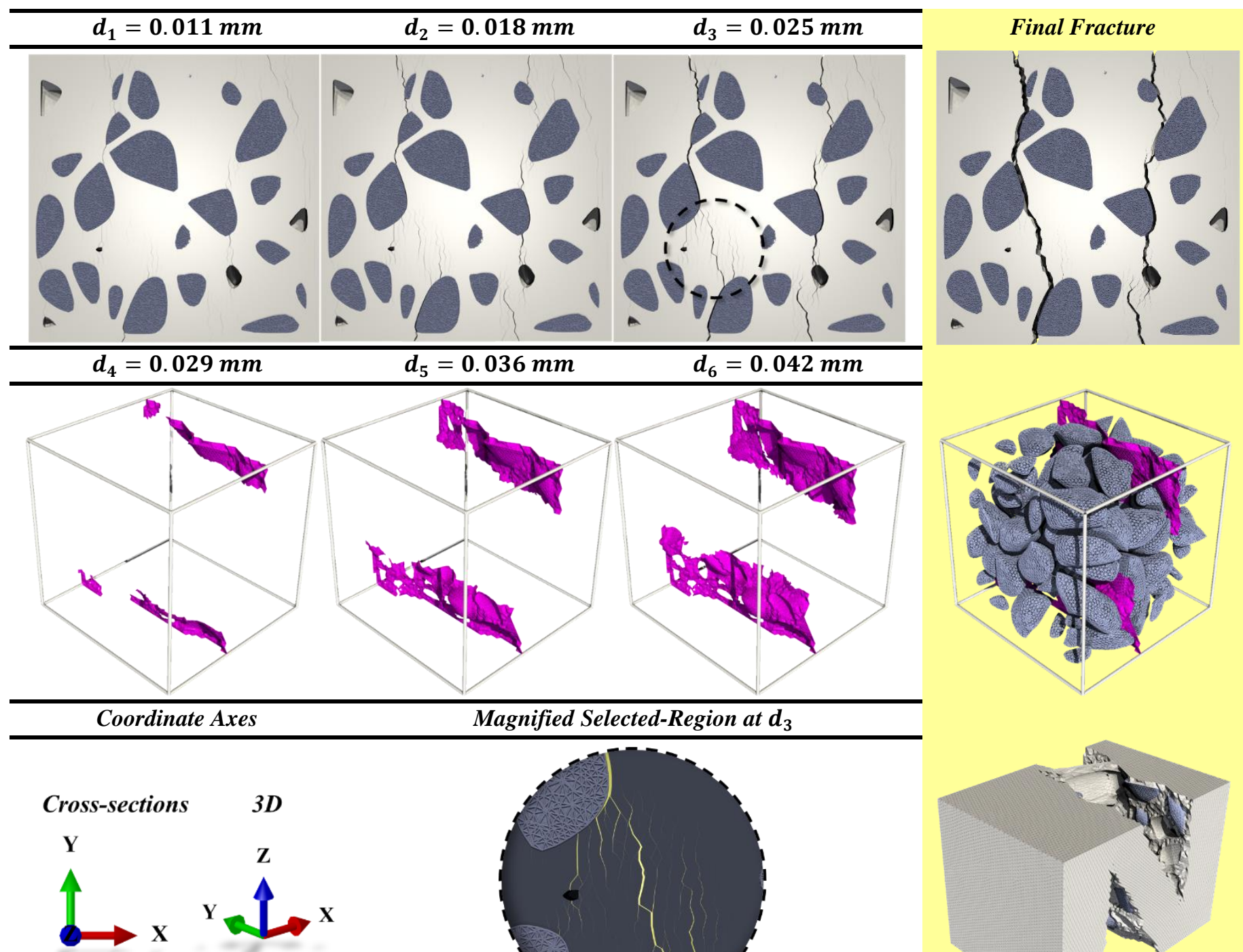

\section{Magnified Selected-Region at $d_{3}$}
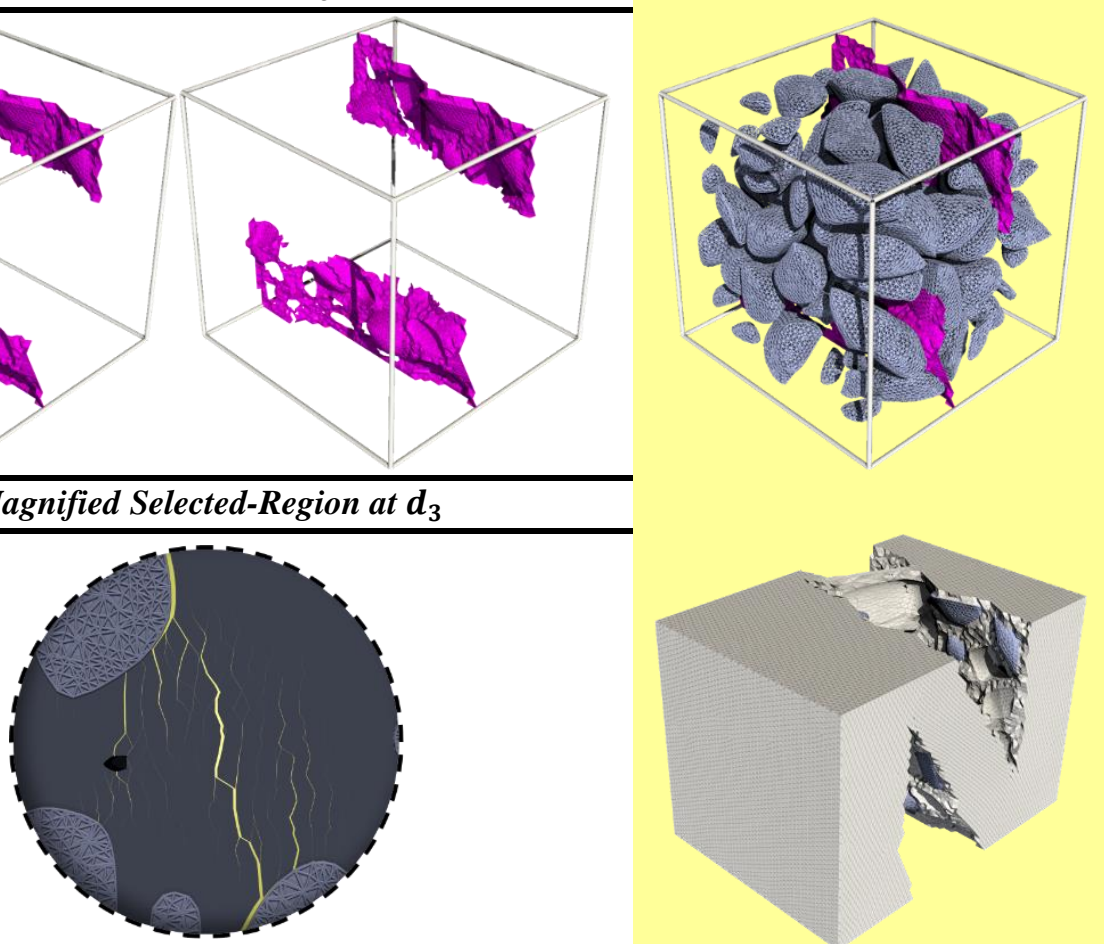

Fig. 8. Visualisation of damage evolution up to final failure in the model IRG-30-0. 


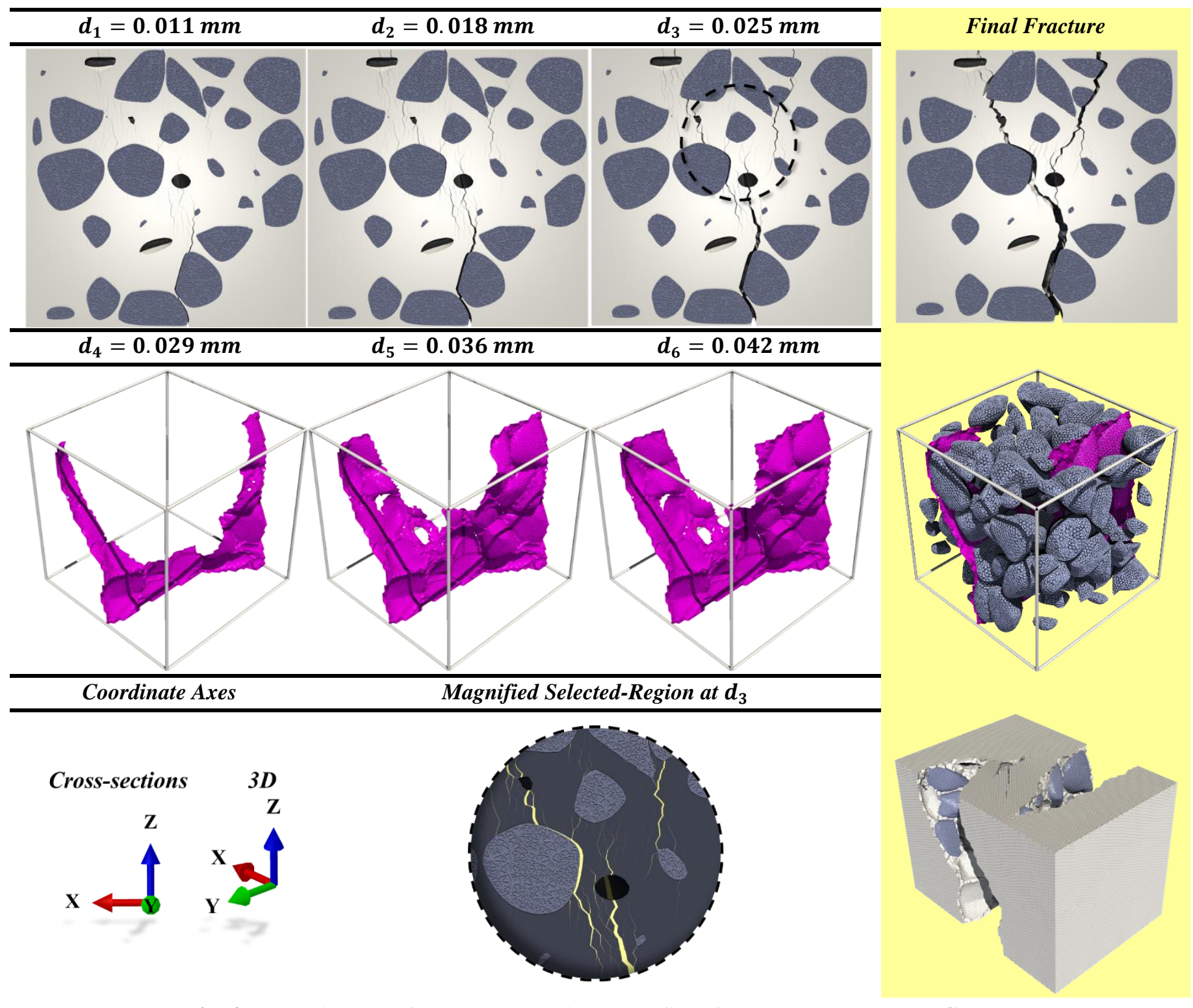

Fig. 9. Visualisation of damage evolution up to final failure in the model IRG-35-0. 

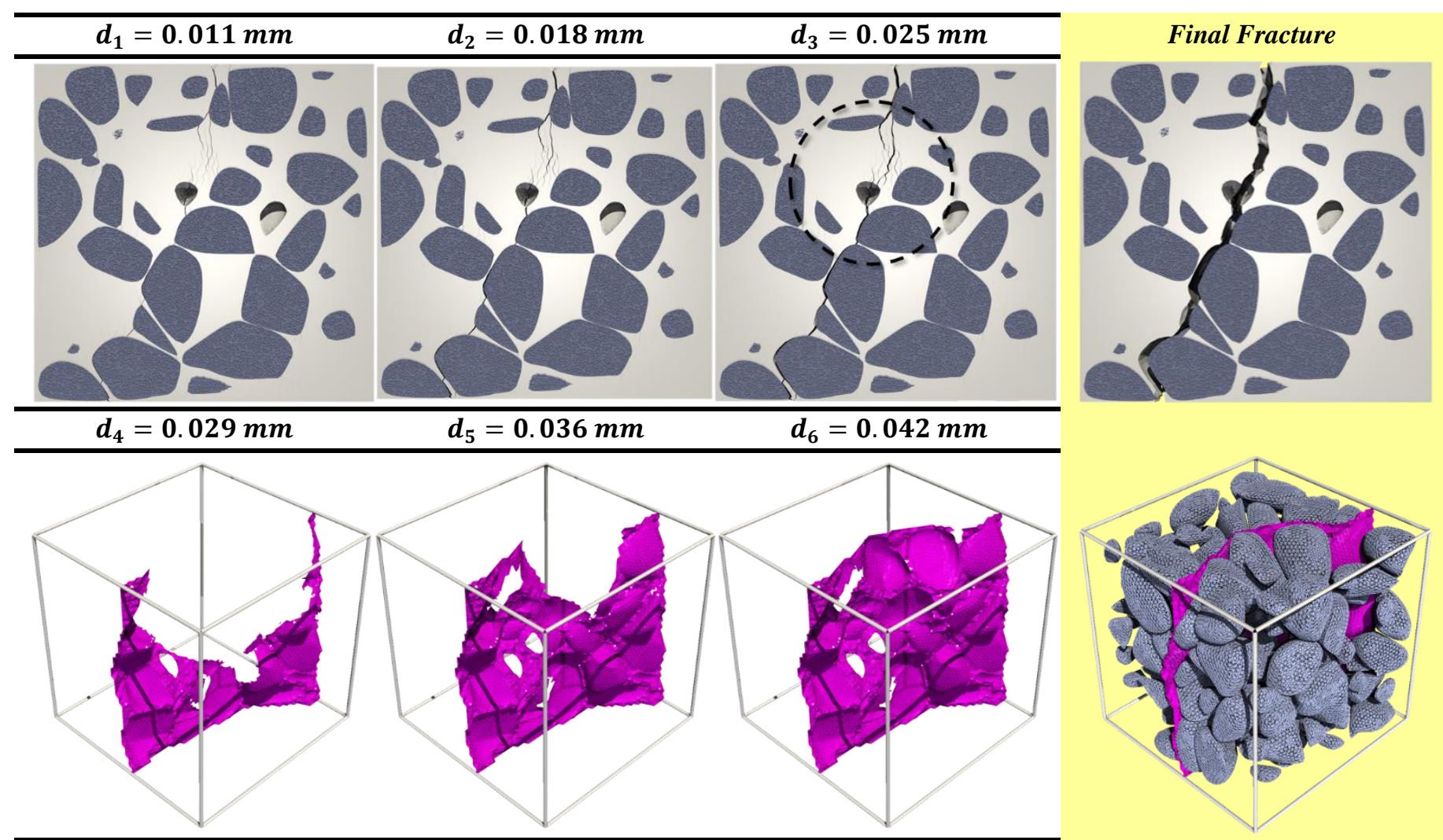

\section{Coordinate Axes}

Magnified Selected-Region at $d_{3}$
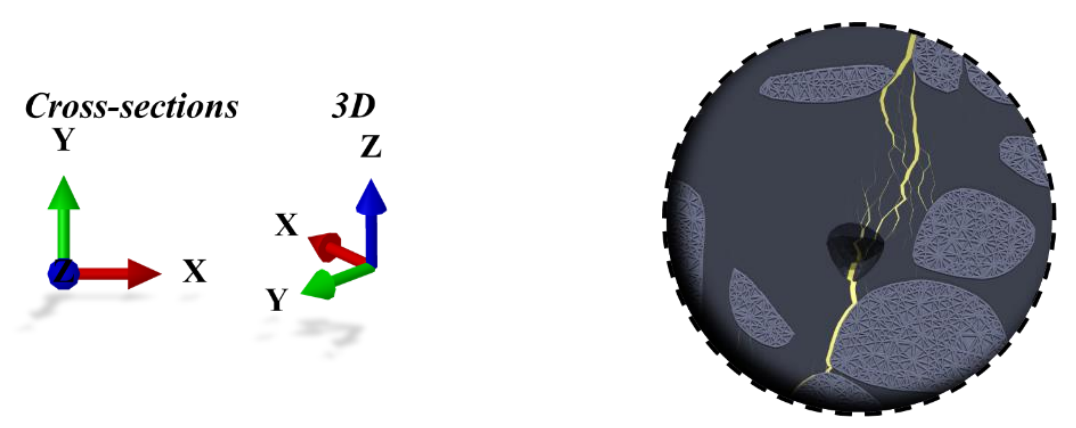

Fig. 10. Visualisation of damage evolution up to final failure in the model IRG-40-0. 


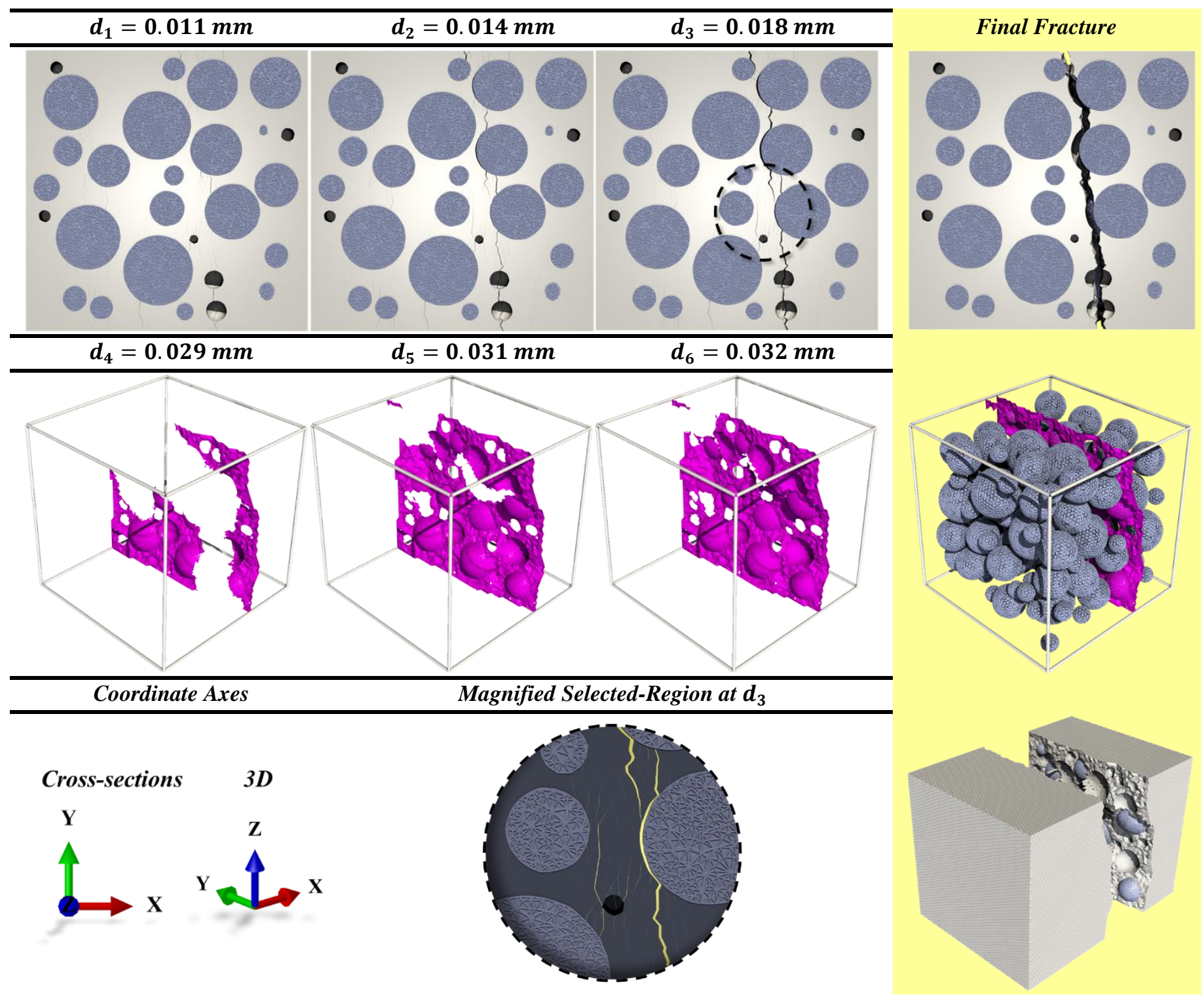

Fig. 11. Visualisation of damage evolution up to final failure in the model $\mathbf{S P H}-30-0$. 


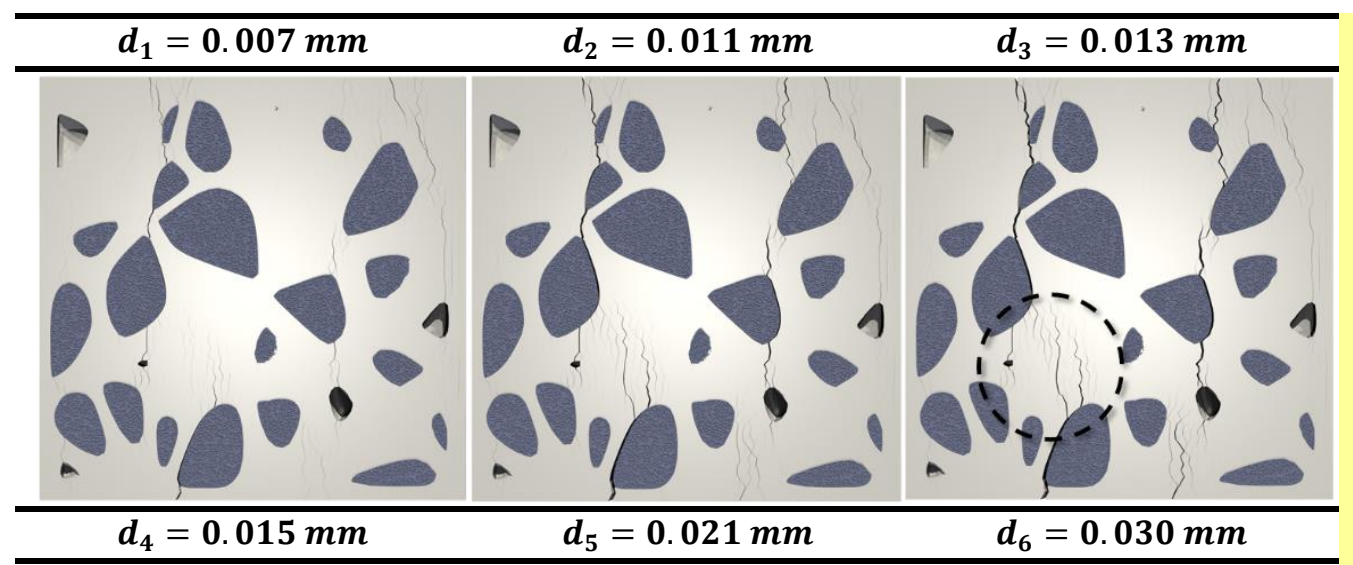

Final Fracture
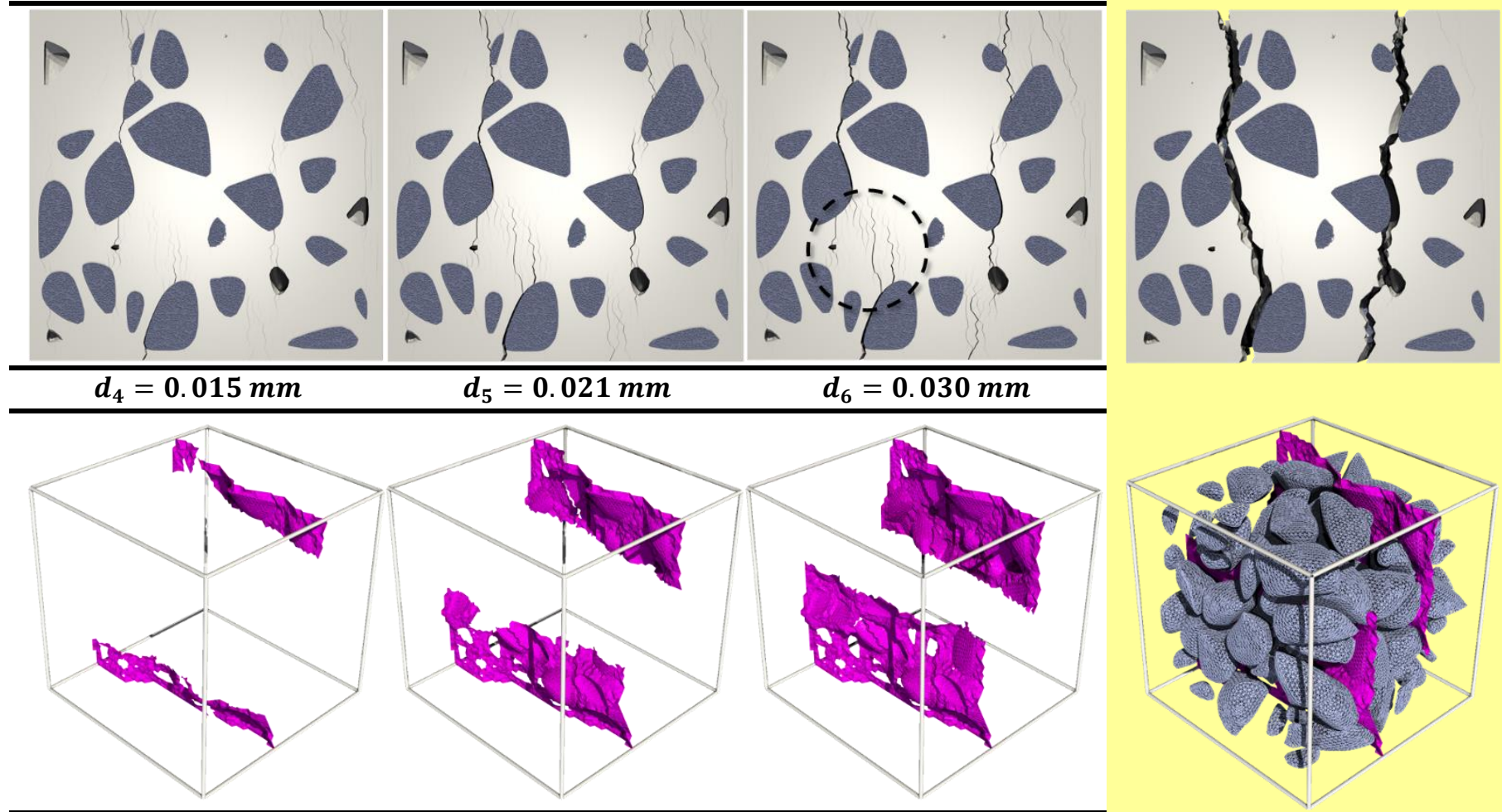

Coordinate Axes

Magnified Selected-Region at $\mathrm{d}_{3}$
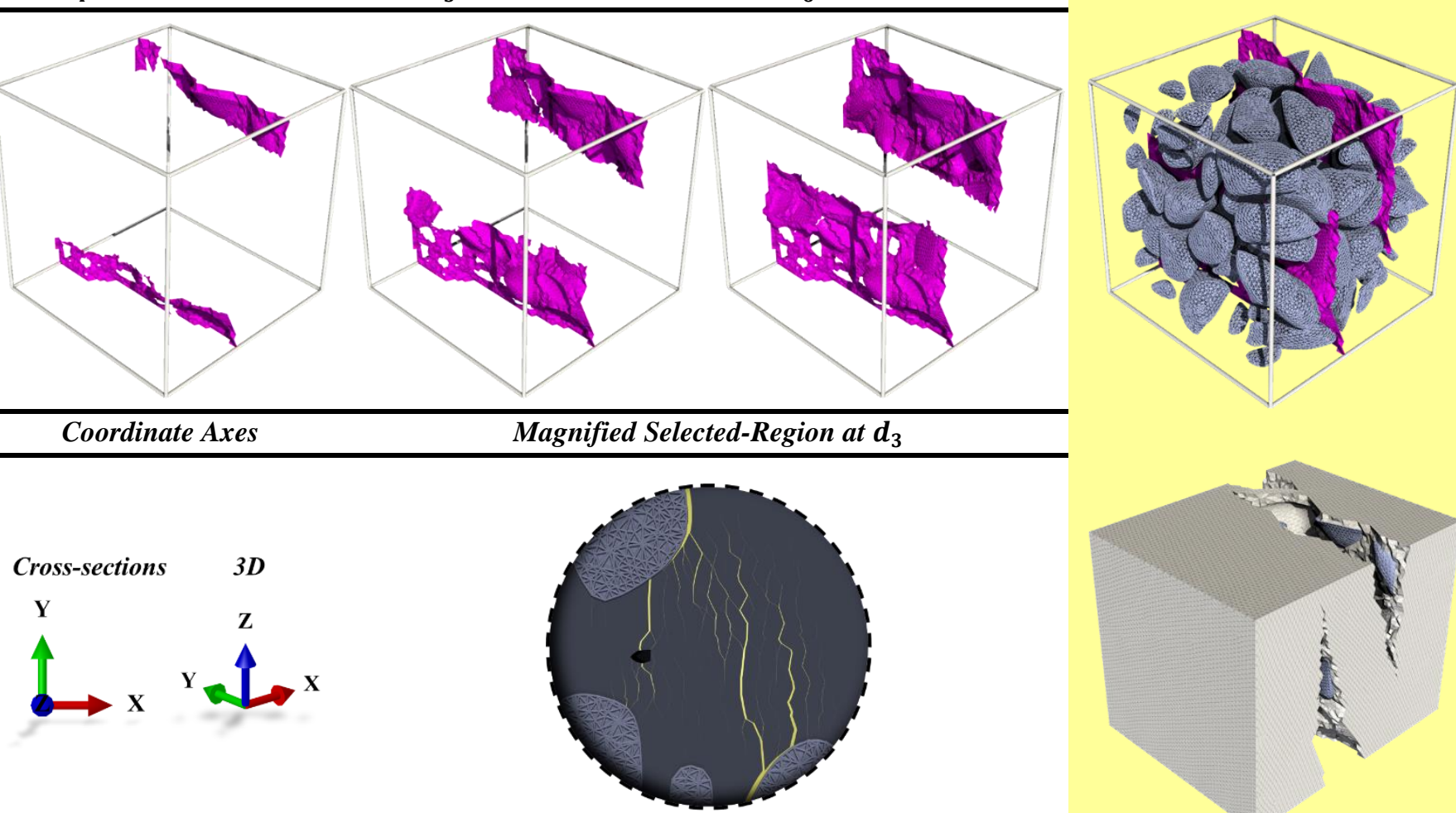

Fig. 12. Visualisation of damage evolution up to final failure in the model IRG-30-1. 


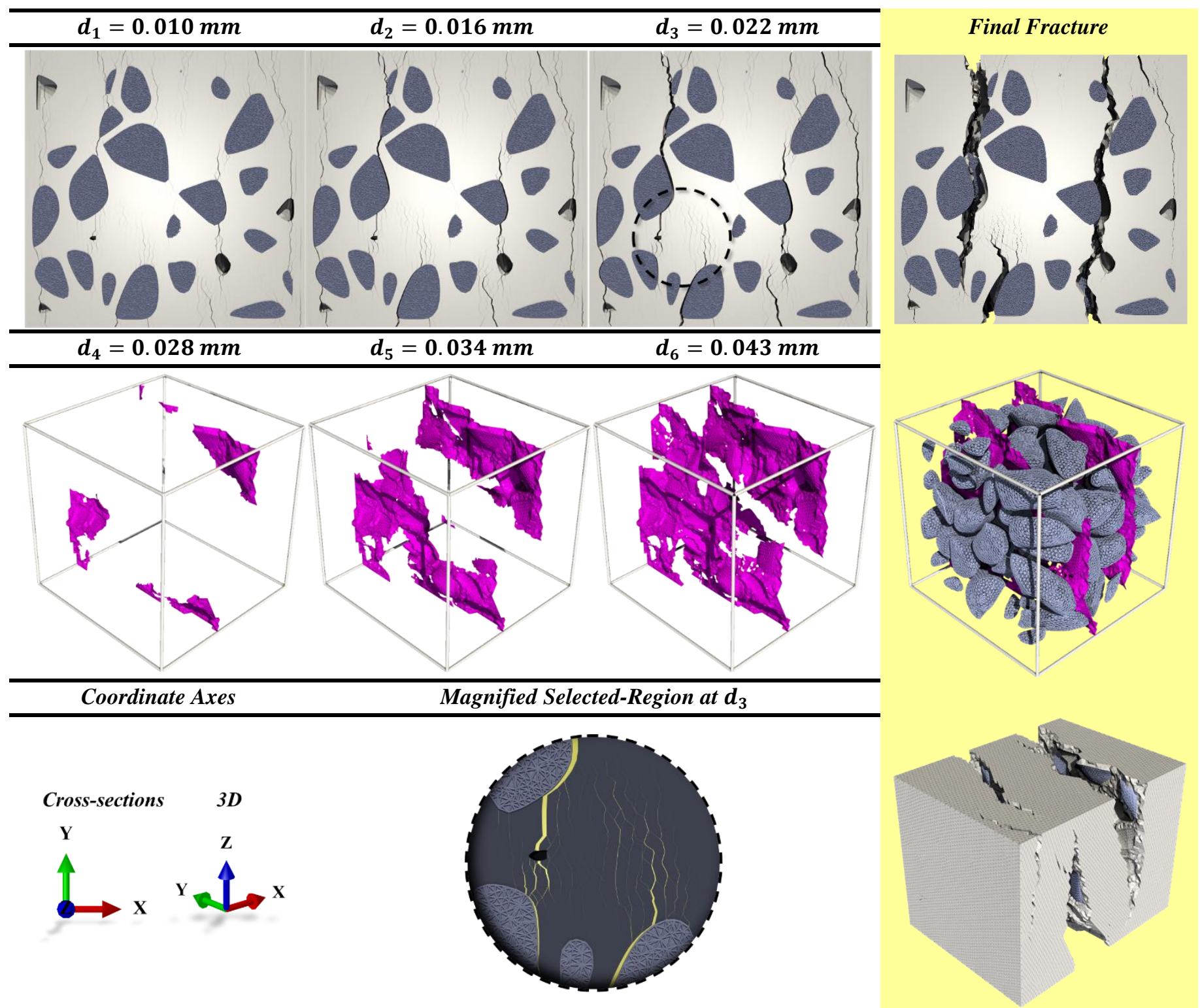

Fig. 13. Visualisation of damage evolution up to final failure in the model IRG-30-10. 


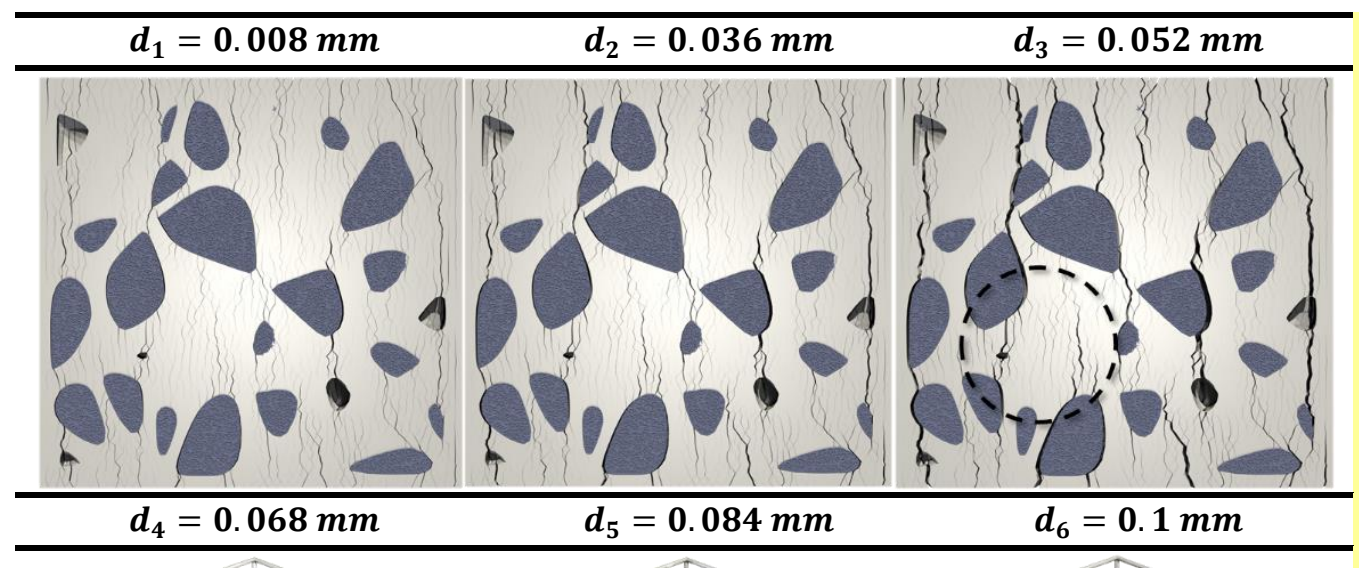

Final Fracture
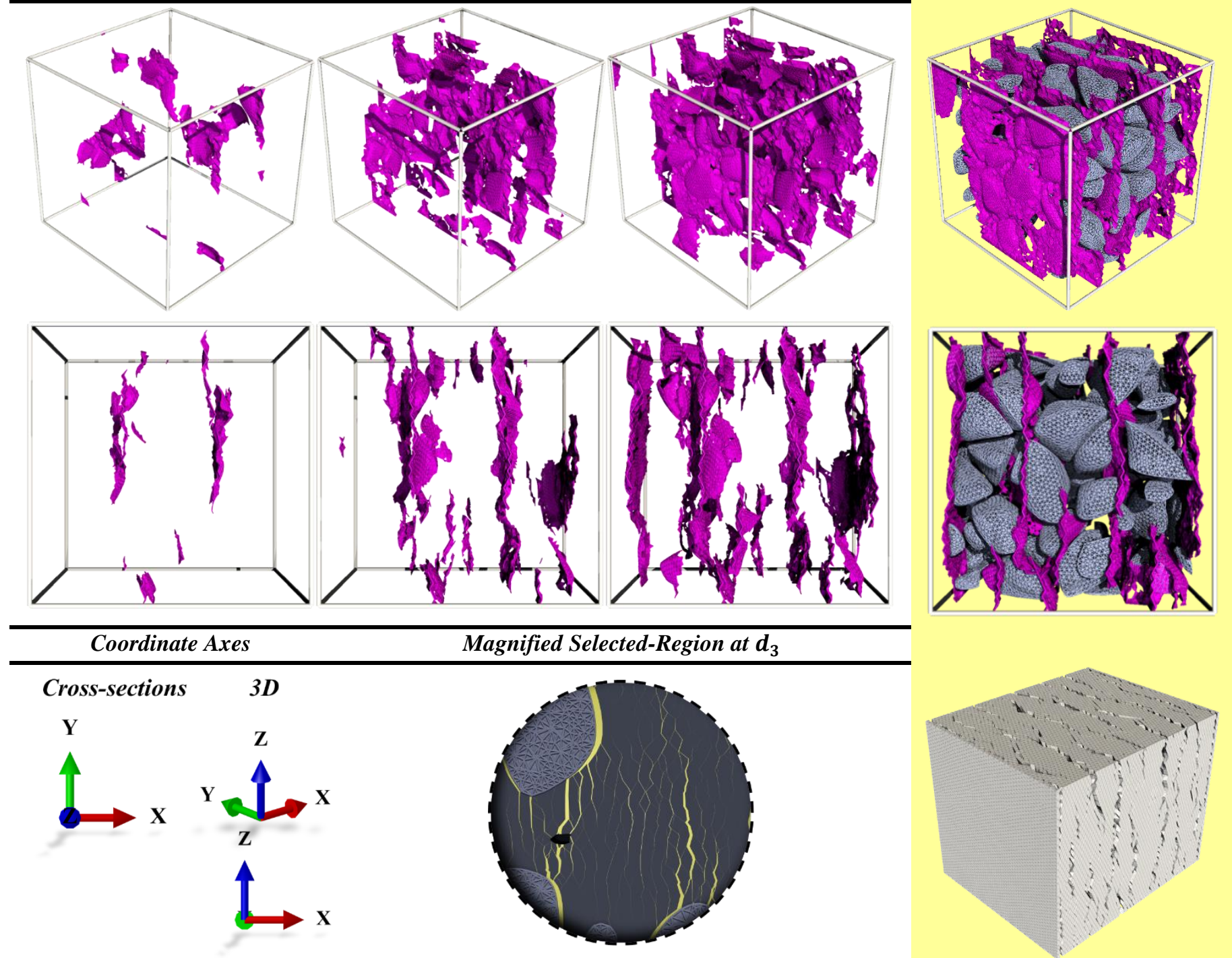

Fig. 14. Visualisation of damage evolution up to final failure in the model $I R G-30-50$. 


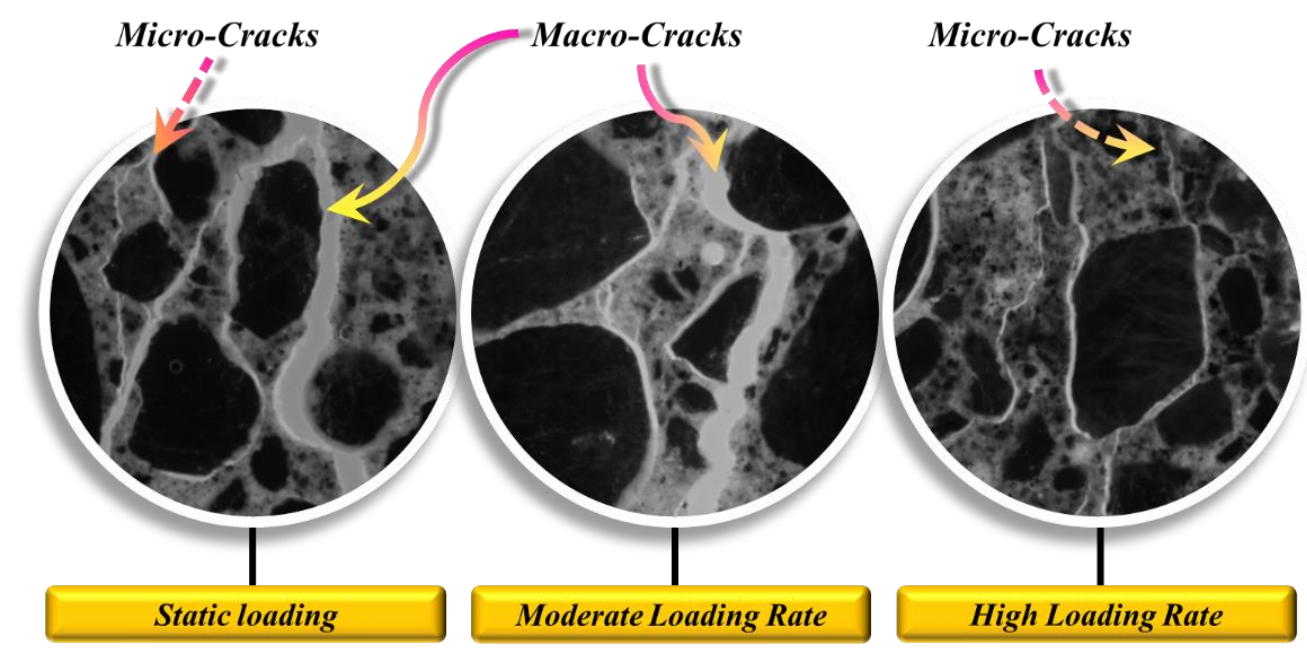

Fig. 15. Micro- and macro-crack patterns observed in the experiments under different loading conditions [67].

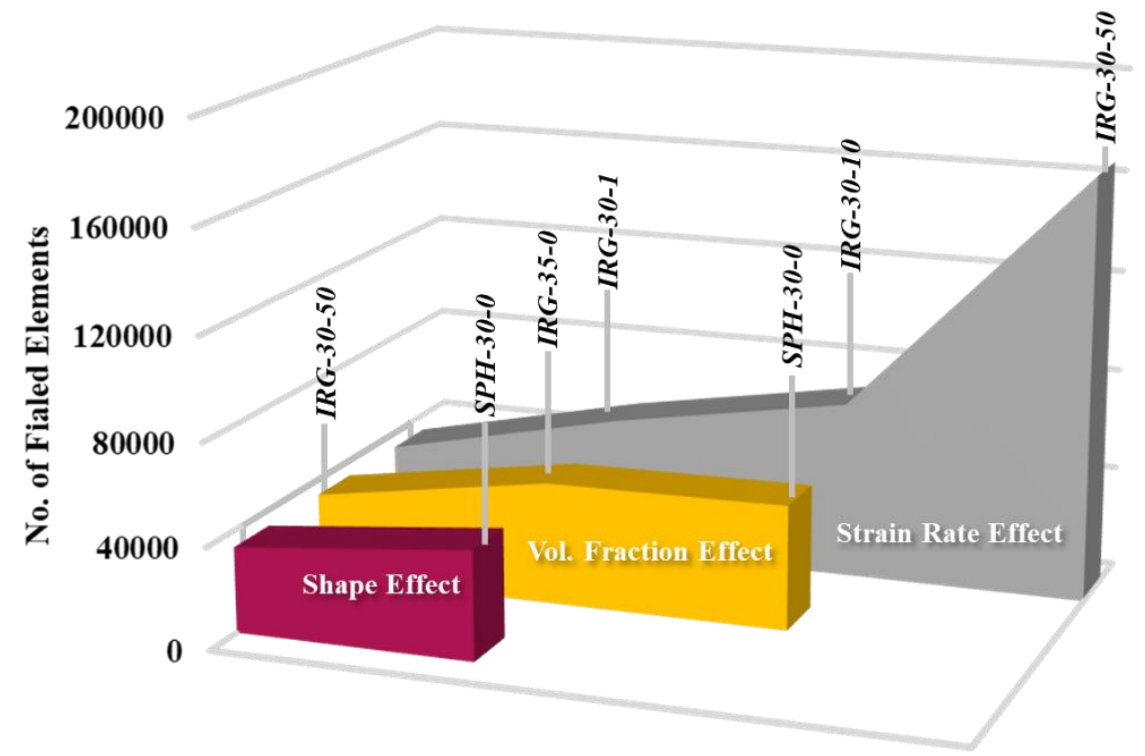

Fig. 16. Number of the failed CIE elements (i.e. $S D E G=1$ ) for each model is presented. The data points are connected based on Fig. 7. 

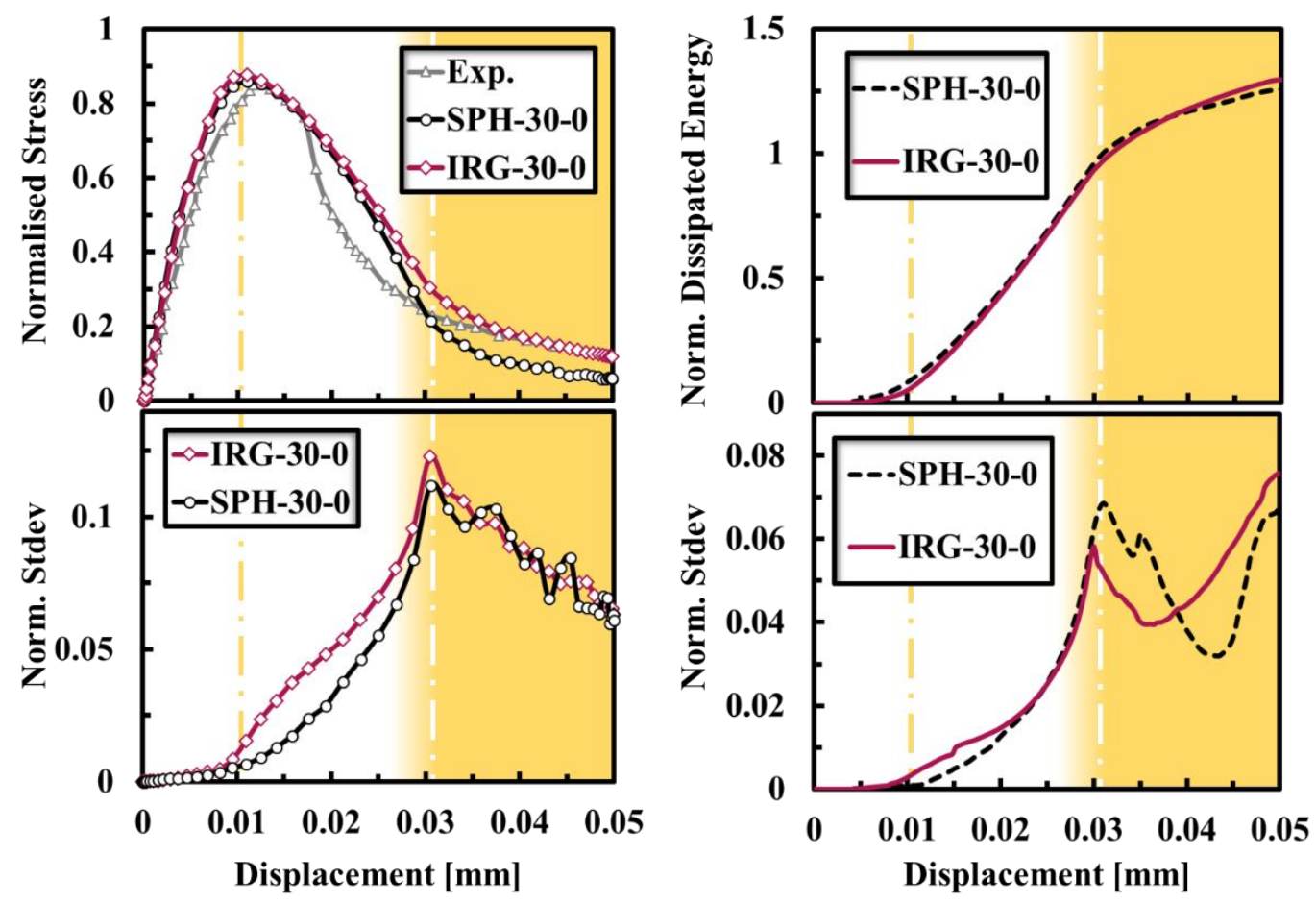

Fig. 17. Stress and damage dissipation responses during fracture process for the models $\boldsymbol{I R G}-30-0$ and $\boldsymbol{S P H}-30-0$.

The stress-displacement curve obtained from the experiments [68] under static loading is also depicted.
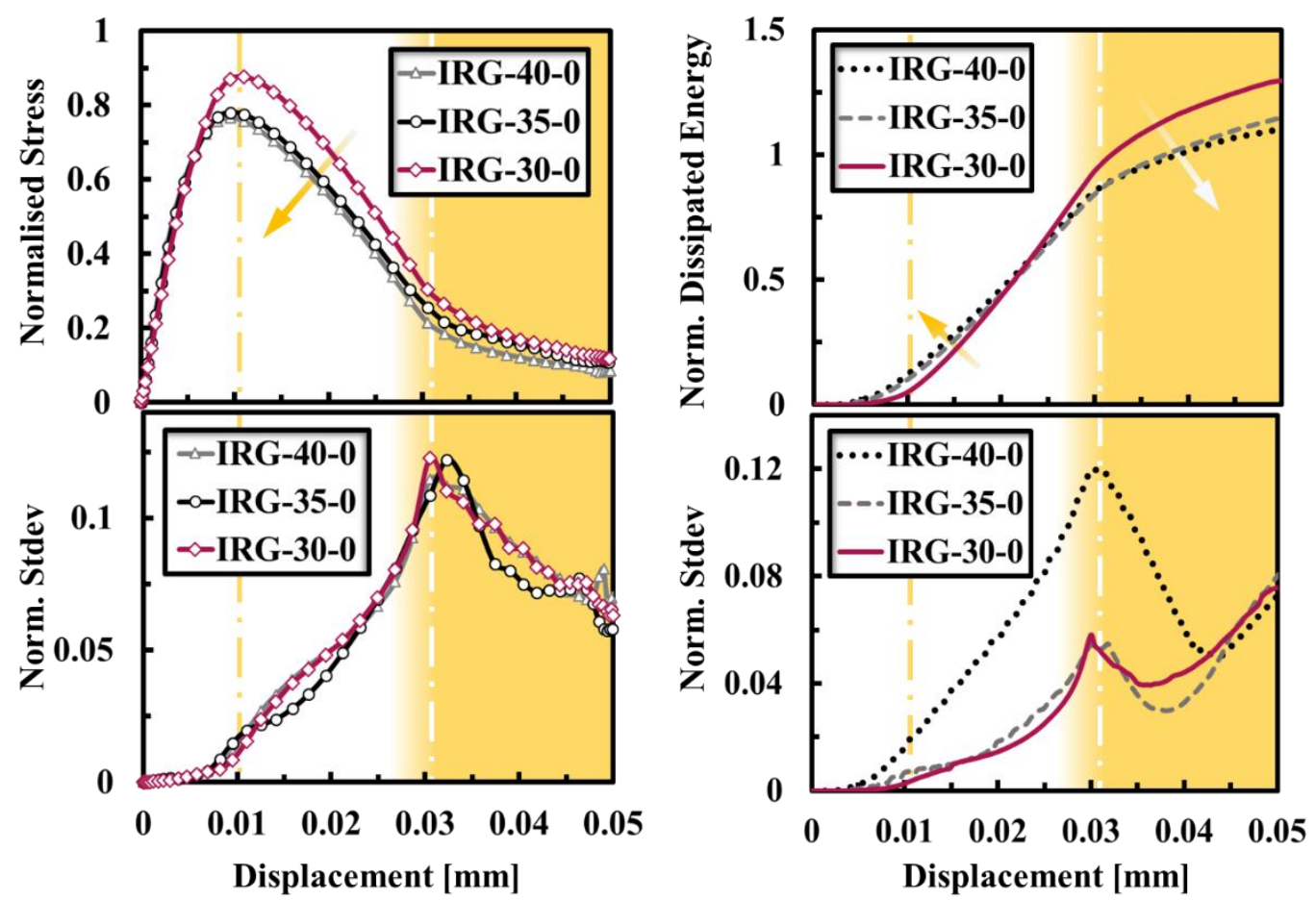

Fig. 18. Stress and damage dissipation responses during fracture process for the models $I R G-30-0, I R G-35-0$ and IRG-40-0. The arrows as guides to the eyes show the curve tendency with respect to the volume fraction increment. 

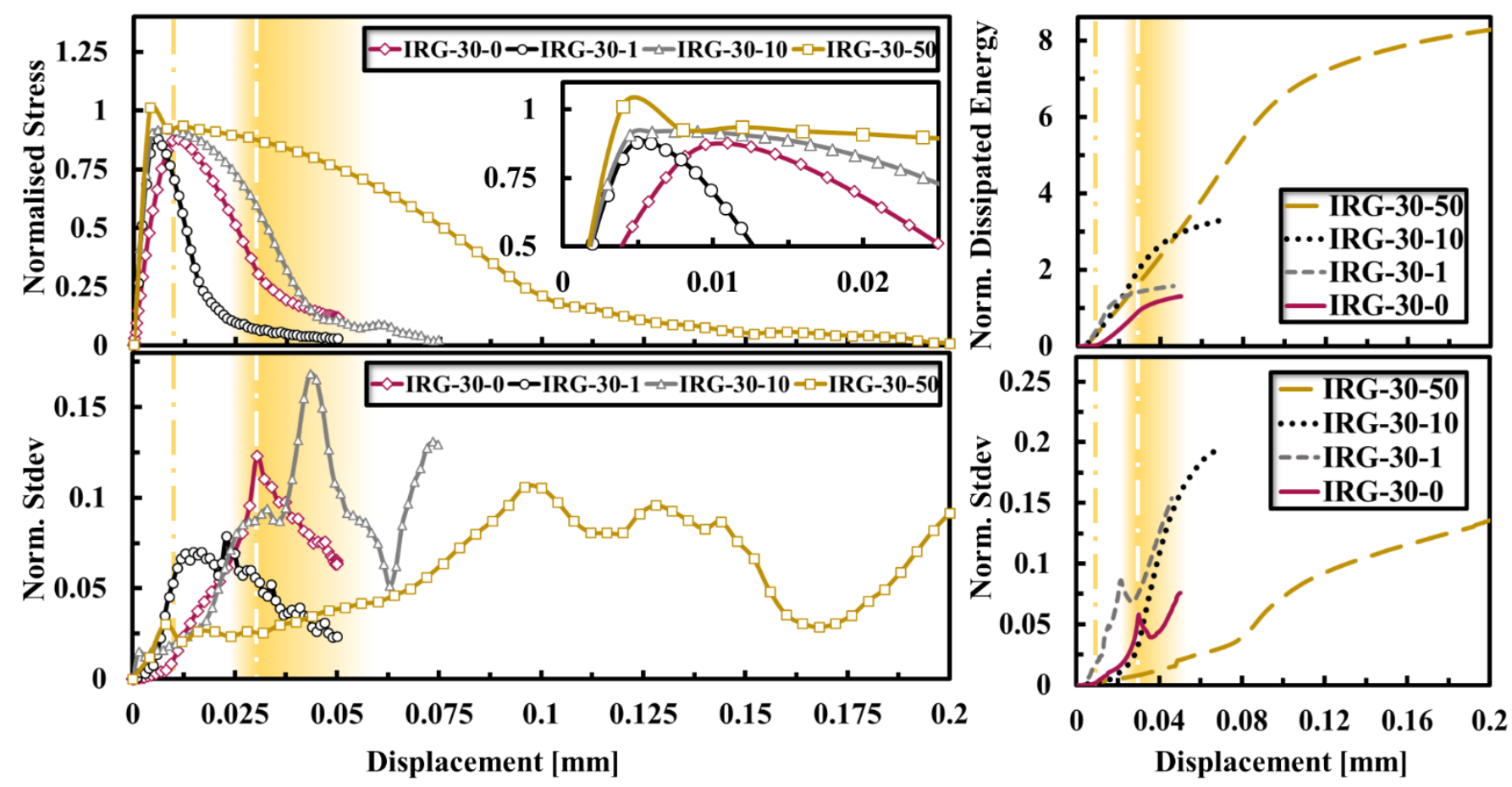

(a)

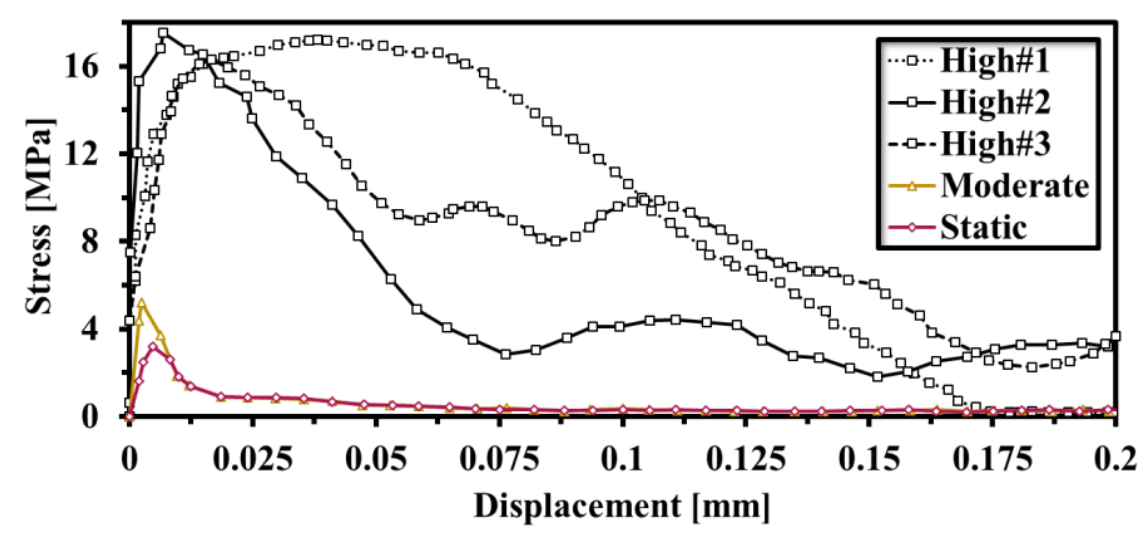

(b)

Fig. 19. (a) Stress and damage dissipation responses during fracture process for the models $I R G-30-0, I R G-30-1$, IRG-30-10 and IRG-40-50. (b) Experimental results [67] for the samples tested under static, moderate strain rate and high strain rate loading regimes. 


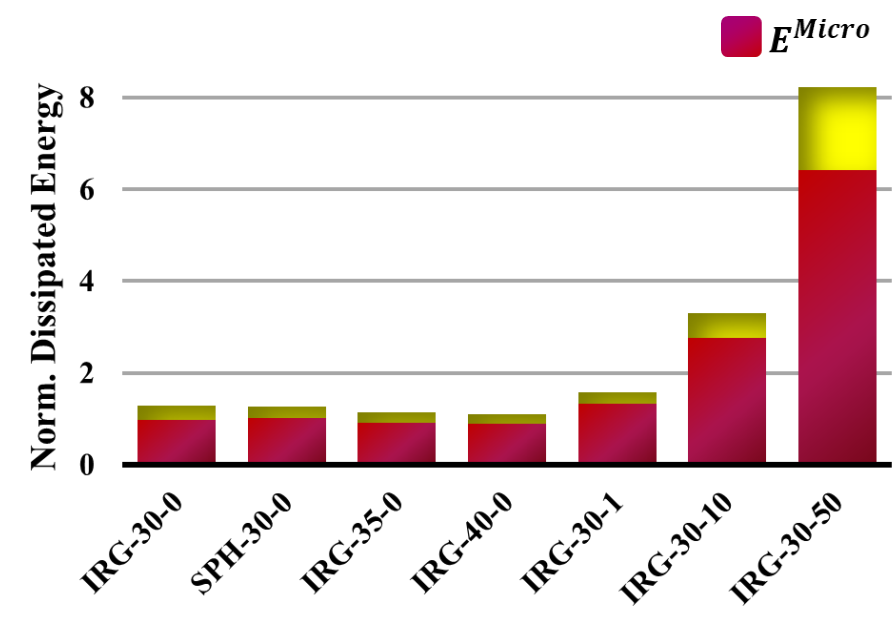

(a)

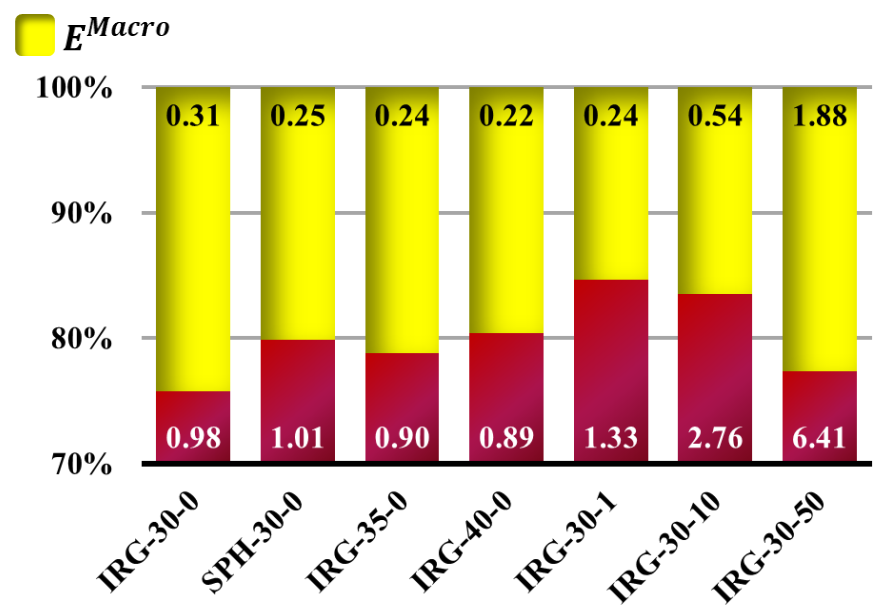

(b)

Fig. 20. Total dissipated energy $E^{\text {Tot }}$ for each model represented by the stacked column chart (a) constructed by $E^{\text {Micro }}$ and $E^{\text {Macro }}$ whose relative percentages with respect to $E^{\text {Tot }}$ are illustrated by the $100 \%$ stacked column chart (b). To ease readability, the values of $E^{\text {Micro }}$ and $E^{\text {Macro }}$ are also indicated on the chart (b). 
Table 1. Particle size distribution of coarse aggregates in concrete [52].

\begin{tabular}{lll}
\hline Sieve size $(\mathrm{mm})$ & Total percentage retained $(\%)$ & Total percentage passing $(\%)$ \\
\hline 12.7 & 0 & 100 \\
9.5 & 39 & 61 \\
4.75 & 90 & 10 \\
2.36 & 98.6 & 1.4 \\
\hline
\end{tabular}

Table 2. Material properties []].

\begin{tabular}{|c|c|c|c|c|c|}
\hline Material properties & Aggregate & Mortar & CIE_AGG & CIE_INT & CIE_MOR \\
\hline Young's modulus, $E(\mathrm{GPa})$ & 70 & 25 & - & - & - \\
\hline Poisson's ratio, $v$ & 0.2 & 0.2 & - & - & - \\
\hline Density, $\rho\left(\mathrm{kg} / \mathrm{m}^{3}\right)$ & 2800 & 2000 & 2800 & 2000 & 2500 \\
\hline Elastic stiffness, $k_{n 0}, k_{s 0}, k_{t 0}(\mathrm{MPa} / \mathrm{mm})$ & - & - & $10^{6}$ & $10^{6}$ & $10^{6}$ \\
\hline Cohesive strength, $t_{n}, t_{s}, t_{t}(\mathrm{MPa})$ & - & - & - & 2 & 4 \\
\hline Fracture energy, $G_{f}(\mathrm{~N} / \mathrm{mm})$ & - & - & - & 0.03 & 0.06 \\
\hline
\end{tabular}

